\title{
RECORD
}

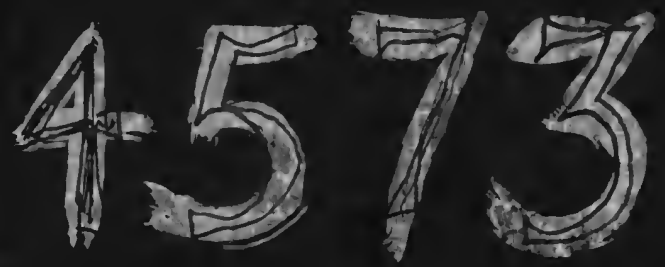


CRPT

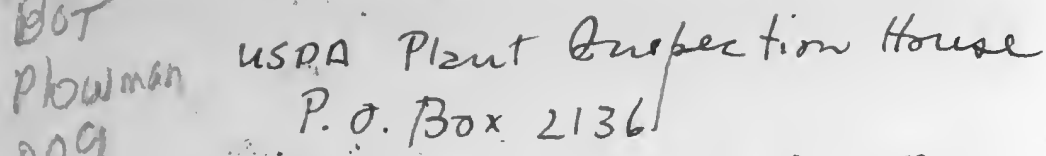
009 MIAMII, FLORIDA 33159

Permit \#37-41840

4

IKT.

PERSONAL

ACCOUNT BOOKS

No. 2044

Available Rulings

JOURNAL

CASH

S. E. LEDGER

RECORD

D. E. LEDGER

144 PAGES

U S. A. 
Flora of Perm $\overline{x \cdots}$ Pant $\overline{1}$ No.2.

p. 427

63. Centapogon wrebanbae E Wt. Wunm. Ropert. Sp. Nov. 38:7 1935,

Q glabruns shrut with dunbug, stender, pendent branchas; leaves oblong, $4.5 \mathrm{~cm}$. Fun , $1.5 \mathrm{~cm}$. corde, acute, nanowed th petwle $1 \mathrm{~cm}$. Iong, flat-crenate, unterspersed with callous to th; inflrescence racemose, lax, long; bracts lanceslate, petwled; pedicels $3 \mathrm{~cm}$. Iong; hypanthium depressed-ghobose, 7 mur. in deancter", sepals livear, $6 \rightarrow \mathrm{mm}$, long, divarecate and towerd the afeex again cscendui; Cosolla Crmson 33 Mum. Iorg (forma); flamentigrabrous; arther tube $7 \mathrm{~mm}$. lona) Mac-pulose m the pussures. 680 - $2050 \mathrm{~m}$. thickets

$63 a$ C. u, bar. estrellanus E. Wumm. Repert. Sp. Noo. $38: 8 \quad 1935$

Louves bradly elleptic, $10 \mathrm{~cm}$. Iong, $7 \mathrm{~cm}$ inde, roundes at the apex, at the trase brwally cuneate to a petiole 15-20 mum loug; pedicels $10-15 \mathrm{~mm}$. Iorg's coroll a sorme shat sueller.

Qyacencho: - dense forest $500 \mathrm{~m}$. 
HELIYHR, A.G.L. Garden vants in cololir... Collingricge 2908)

HUIJYIR, A.G.J. The encyclopedic of plant purtaits (Coilingrides i953)

JAIRSON, H.G. IIlustrated aride to the trees anj flowers

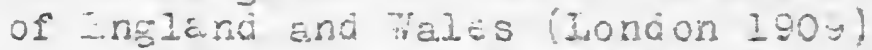

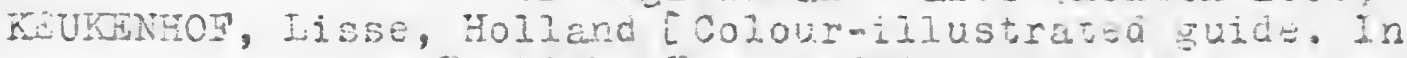
inglish, In., and Germen snd Diach. n.d.J

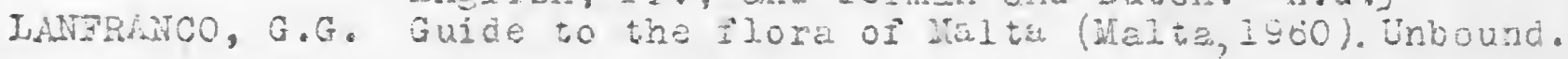

MATSFITID, T.C. The border in colour. Revised ed. (Collins 1947)

MiRTIN, "K. The concise 3ritish plora in colour...2ne ed.

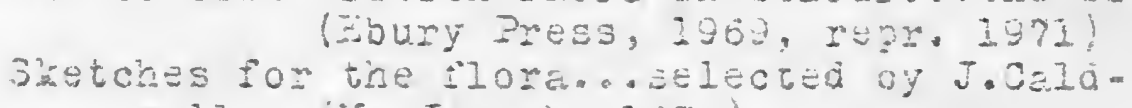

MLRTIN, W.K.

SAIDEES, ก. T.

SFRY, C.

SPRY, C.

SYYGE, P.Y.

WAGGIRI, $Z_{-} \mathrm{H}$, vell... Jir. Joseph, İ72)

Rock gemdors and alpine plants...3ri ed.

Flower decozation (Dent 1934, regr. 1947)

How to do the flowers (Ataritis Eubl. Co, Iondon, c. 1945?) Unocund.

Colins guide to oulbs (Colinis, 196j)

Die schönsten Alpenblumen (Vtrein zum Schutze d. Alpengflarden und - Tiere, liunchen) 


\section{Lemna ecuadoriensis sp. nov.}

Lemaie obscurae valde affinis sed differt frondibus angustioribus (1 $1 / 2-2$ partibus longioribus quam latibus), radicibus brevicribus. Fructus incogriti. Habitat in Ecuacior.

Type collection: Ecuador, Prov. El Oro, between Machala and Santa Rósa, $300 \mathrm{ft}$; in a drainage ditch. leg. T. Plowman, L. Jacobs and E.W. Davis 4609; Decembër 2, 1974. Holotype: U. Isotypes: F, GH.

$L$. ecuadoriensis is very similar to $L$. obscura but its fronds are generally narrower, 1 / $2-2$ times as long as wide ( $L$. obscura; 1 1/5-12/3) and the papule at apex seems to be still more prominent; the fronds are dark red underneath and the roots relatively short (up to $1,5 \mathrm{~cm}$ ); flowers and fruits are not known.

The species is solely known from the type locality in Ecuador; it is therefore difficult to decide which features are diagnostically relevant and how large the whole variation specter is. The taxon might be considered as a variant of $L$. obscura; however, the next station of $L$. obscura is more than 2000 miles apart in Mexico and climatic conditions at the type locality are different from those occurring within the main distribution area of $L$. obscura. The climate at Machala (Ecuador) is warm throughout the whole year and shows a very pronounced dry season; on the contrary, the climate of Southeastern North America where L. obscura has its optimum is cool during winter time and has a high rainfall during the vegetation period. It seems reasonable to describe this collection provisionally as a separate unit. More material from the region is desirable.

E. Landolt in Veroffentlichungen des Geobotanischen Institutes der Eidg. Techn. Hochschule Stiftung Rtbel, zturich. vol 70: 22. 1980. 
TIMOTHY PLOWMAN BOTANICAL MUSEUM HARVARD UNIVERSITY

CAMBRIDGE, MASS. 021:8 

ECundor: Pror. Chimborazo. Rosd from Riobrinba to Guamote, Alt. $9800 \mathrm{ft} .28$ nor $197 \%$ with Wade Devis + Lee Jecobs, 4573 . Acnistus $=\frac{\text { Iochroma }}{\text { Lehmannii Bitter }} \mid$ A.Hunzinger 1983. usmline Tree $10 \mathrm{~m}$. tall along strean, Mo kis trme $30 \mathrm{~cm}$ DBH. Corllla is greenis yellow. FRs. pale greer.

(A) $>K$

Cit 457 . mouning

(T) 2) shrub $1.5 \mathrm{~cm}$. Corllo danh. blue, keel yellow. FRs, reddifl.

4575 . Bomarea

Cit (1) Vine in thichet. Caly + corrlla aank red abaxially, pale yellowijh red adaxially

4576. Bomerea

U. (A) (3) Sine in thicher. Sepals orange. GIt, b2xisily, scumer green, yellow sdaxidy. $S_{A \rightarrow G B}$ Perals qolder yellom abaxisly, with

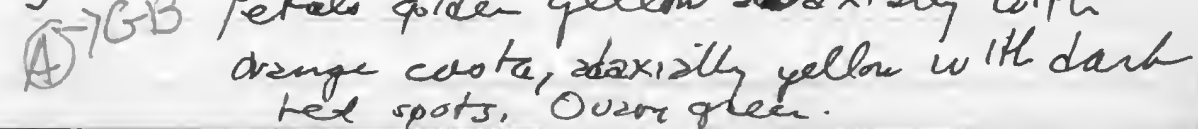


Git 4577. Podocarfus

$s$ (2) Shicher or tree to $4 \mathrm{~m}$. i

Git 4578 . Sisgrosanthus Chimboracenis. U. (2) trent in pastiche ar hellads. soed PTBC Fis. light blue.

GH 4579. Stenomesson aurantiacum Amaryllid.

(A) (1) Bulbiferous ferb. Orany gree.

Fij. orainge.

(A) $\rightarrow K$

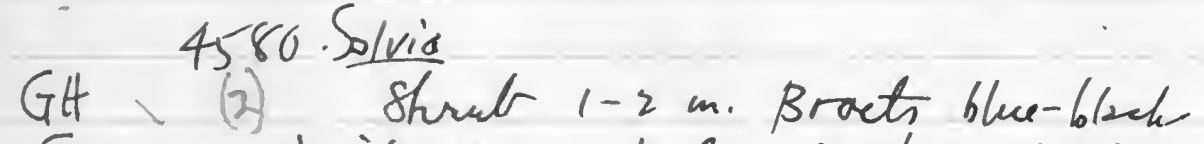
$S$ spicily. green below. Crolla dasb

458! Bomarea

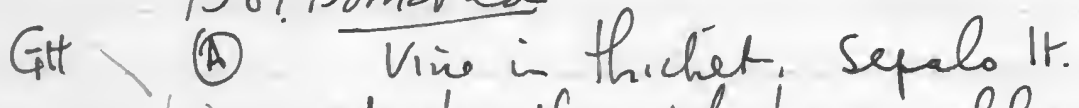

(1) red abaxiale, pale bmm-yellow, adzxiath. Petals yellow-green', 2 oxizty with medial red stripe, adaxidy with targe brown splotches (A) $\rightarrow K$ 
4582. Benteris

Git (2) Shrub $2 \mathrm{~m}$. in thichat. Buds $s$ red-orange. Tepalo boight orange.

Oreocallis mucronata $\left(R_{i}+P\right.$. Sleumer 4583. Embothrium det B. Sparré76 Proteacen USM (6) n.v. "galusy" - fis; for kidney ail ments. Fol reeds 3 Shrub or tree to $6 \mathrm{~m}$.

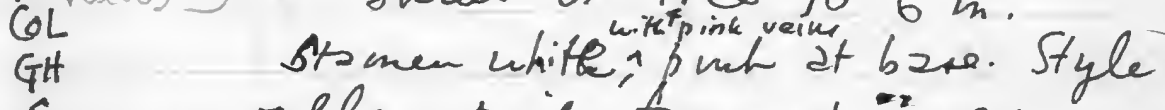
$s$ prpirgelow, pind fowand apex. seed pribr Stigma yellow grea. det C. Niezgoda $19 \%$.

(4) $\rightarrow K$

458\%. Chuquiaga Comprite Citt 1 Brange. Corrlla yellow. Styles des orauge.

ECUADUR: PROV. CANAR.

1 Dee 1974.

4585 . Erythroxylum

Econ (1) "Coca"

Cultivated at Cochanciay.

Provided by Interpol Agent Capitar guillermo gomezjurado. lange If.

Econ 4586 . Erythroxylum

(1) Cultivated at Chilcales.

sminleaf. 
Ecuador: Prov. AzraAy.

Rosd Cuenca to Jiron. 1 Dee 1974.

A It. $8020 \mathrm{ft}$.

4587. Streptosulen jamesonii

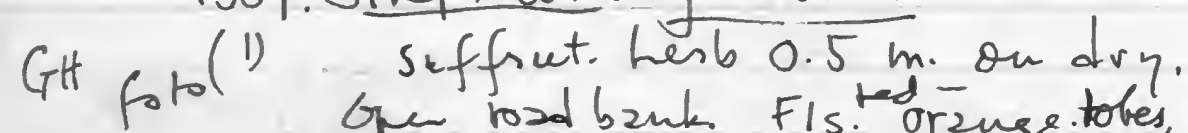
Gew rosd bank. FIs. Orange tobes,

4588. Pitcaimea pavonii Mez Brmelese.

U. for (3) terb on steep rodbouk. Brats greer. Cal $\times$ pale ted Corrua deep red.

Alt. 6700 fl. Rosd Jiron-Catavita.

4589. Passif lora manicata (Juss.) Pers.

Glt

Vine in theket. Bracts baslly

(2) (A) light gree, apicilly bromish.

5

Pedical t hypenthin pole gree. Seple pale green abaxisly, red adaxiclly. Petals light réd abaxialy.

sed PTBF. bright ted adaxially. Corma pusple with white sictaids edge. Gyuopleore pale grea. Fr. gren,

det. P. Matekait is 1982 .

(4) $) K$ 
Prol. AfuAy

Alt. $5500 \mathrm{ft}$. Gatavita-PASAJE:-

4596. Tropadem minus $L$.

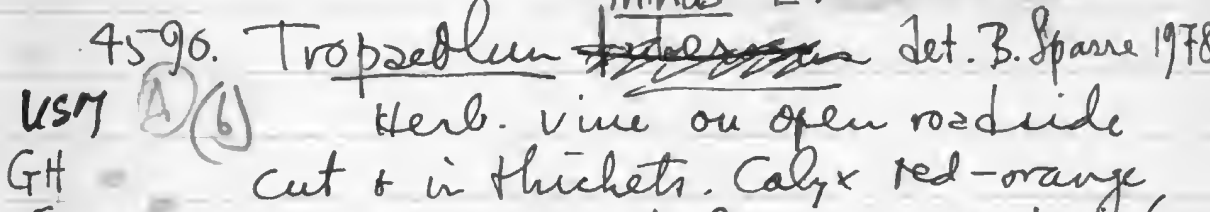
brver 2 lobes 5 brver 2 lobes pale preenish, trpe's us green. Upperiz petals yellw. RHswislen orange with this ied striper. Lower 3 WTBKT petals clacued, yellow-orange with red orange spot adaxiolly'. Firs. pale green.

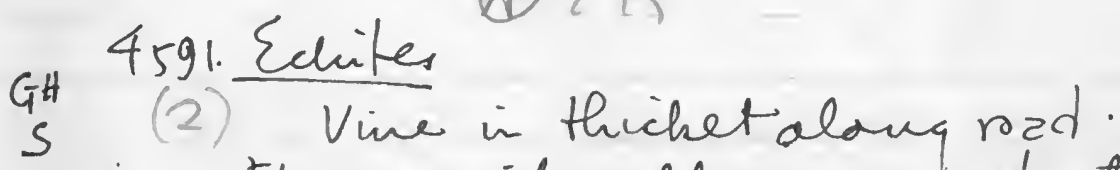

(4) $\rightarrow \mathrm{K}$ FIs-greenial yellow, margin of petel bright yellow.

4592 Trichocereus pechanoi

GH (I) Cactus $5 \mathrm{~m}$. tall on steep on hillside. Hypanthien tabe green With blach indument. Outratepels hiptir green, inner tepals white. det. m. Flones 1979 .

$\mathrm{GH}$

4593. Peperomia $S$

Succulent herb on dry hillside. Spither red. 


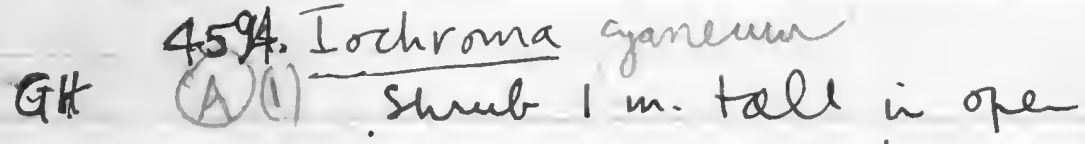
waite place along road. Calyx dark green. Corolla deep purple. Frs pole green purplish tinged.

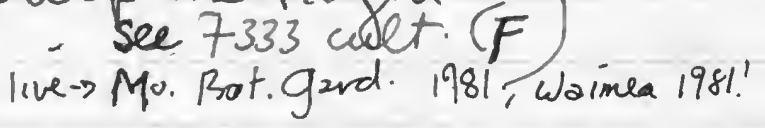
Alt. $5100 \mathrm{ft}$. Km. 96. from Cuenca
or road to Pase.

4595. Nicotiana glutiursa L.

GH (3) (A) Sticky herb Imo tall on 5 dry roadside. Corolla tube yellow BUR green, lobes pale red within.

Gt $\$ 596$. Opuntia Low creeping er cactus. D vary green. Tepals bright red-oralge, outer teals greenish yellow. $\leftrightarrow K$

4597. Jatrpha GH Hest with succulent stems
Milky Yates. Corse bright
red? 
4598 Euphrrbia weberbaneri Mansf. Euphor hide.

GAD (3) Leaftess branched shrut with 5 yellow green stems. Inf1. $+f$ ls. dark purple. det 9. L. Webite 1975 PROU. AZUAY

Alt. $2800 \mathrm{ft}$. JIRON-PASAJs

4599. Jequemontia corymbulosa Benth. vine on dry hillside along road. Cosolla It. blhe. det K. Robertson f6.

4600. Eucrosia aurantiaca (Baker) Trinb 1981. U. (1) Bulbiterous hes $b$ on steep usy (4) dry hillsides. Pedicil t vary GHt neds I darh greer. Corrlla 1t. yellow, \& $\rightarrow k$ Filaments pilegreen; FRs. $(=7634)$ darh grea. Bull whet.

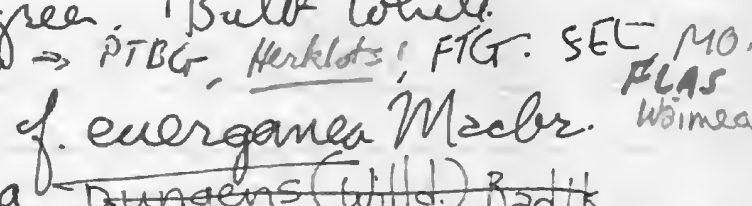

$\mathrm{F}_{S}^{G H}$ 4601. Clavija pungens(willd) Radik. (3)(A) Tree or shrub to $3 \mathrm{~m}$. Fls. seed PTBG orange: FlKS. orange. 4 . Niezgoda 19 fb. 460la Clsvijz energana

(4) $\rightarrow K$ Seedling from 4601 . 18 oct 1978 

$\rightarrow$ J.weberbaneri Helwig det.D. Austi.

\$602. Jequemontio of . quyanensis (Aubl.) Me'suner S seed. 3) Vive on Hadudes. Fls.

Git nuds I light blue. det.K. Robertson ff.

Git 4603. Pisonia

(1) Shrul $3 \mathrm{~m}$. Fres. dark brow.

4604 . Bruhinia aculeata $L$.

GH (2) (A) shrub 3-4m. Corlla pure white. Frs. brown. Colyx pale grear.

GIt 4605. Anthurium pohlii der TBCroot

(1) Epiphyde. Lus. Coriaceous. Spathe light green. Spadix dall plink.

Alt. $2500 \mathrm{ft}$.

GH \$606. Escobadea scabrifulia Herb on ticts ide. Fls. pohite. 
4607. Mounina

GH (1) Kentr Im. Rachi palered. Fis. violet, keel yellow.

4608. Epidendrumen

Ames (2) Herlo on boulder along road. Fis. pele greemist bhom Lemna ecuadorensis Landolt det E. Lomdolt 1978
(3ynion) ECuArork: PR̈́r. EL ORO. $2 \operatorname{Dec} 1974$. 3 ro ft. alt. Between Machale + Sautaloosa. GH-iso 6 . 60 . Lemna perpusilla Torrey

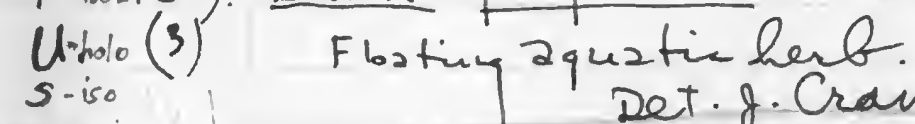
Det.g. Cramer 1976 . Road Arenillas to Huaquillas. Alt. $4 r \mathrm{ft}$. 4610.

GSH

$\mathrm{s}$ vine ain dry deciduous forest. Fls. White.

4611. Carica parviflora (A.DC.) Solms"

CHA (4) Shrub untranched to $3 \mathrm{~m}$. with

(2) semi succulent stem. Rashis f's. bright pink.

46.12. Killandsia disticha H.B.K. Bromeliad, (A) Epiptyte inden thorn sckect. FIs dull yellow det TB Suill'76 
Peru: Depto. LimA. Prov. Ganta. $V_{2}$ lle del Rio Chillon. Yangas Alt. $3500 \mathrm{ft}=8 \mathrm{Dec} 1974$.

4613. Rumer

GHt (1) Weed $1 \mathrm{~m}$. aloug river, i band.

4614. Zanichellia palustris L.

u. (2) Aquatic submerged herb is Ris chillon. det. Etrecut 1182 .

USM 4615 .

Cucusbit

CTH (2) Viuse on rièren bouch. Fis. yellow. FRs. bright yellow-gleen.

USM 46,16 .

CFIt 2 Aquatic hert on shellow place slong rive. Stems red-purfle. Fis. yellow.

4617. Helistropium angio spermum Murr. (2) Herb on riverteoch in sand. Gis Fis. whit.

asM 4618 . Heliotropium arborescens $L$. Foragin. Fis: vale vilet det. M. Frohlich 1984. 
Git 4619. Desmodium

(2) Subvining hent on sandy beach neds 1 aloug riven. Fis. blue-irolet.

4620. Pasifiora suberosa $L$.

UST (3) Herbaceons vine with thich woody. GHt mistack in sendy leach along riven. Perienth It.grean. Corrta yellow.

Let. P.m-Jorgensen 1984.

4621. Cestrum

USM (2) Shrub 2-3 m. 2long irr.getion ditches (THt Conlla tube greenish yellow, lo bex dk. browon.

usm 462z. Aanistus a rborescens (L.) schlecht.

(3) Shrub 1-3m. along irrigztion

Bent ditzles. Fis.creary white. FRs. pole orange.

(A) $\rightarrow \mathrm{KH}$

4623 Gyclartherar pedata (1.) Schrad. $K$ Econ. (3) Cult vine aloug irrigation ditel. Fis. yellowish. Fre green. 
Prku: Lima. La Molina. $24 \sec 197 \%$ Jardi Botanico.

4624. Enthroxy/um novgranateune (2) Tran. Haxillense (Rusby) Plowman $K$ Cult shrub 2 n. tall in sandi Botanico. Buds creamy yellow. From Trujilo.

Peku: Tambo to Vis o-Tamb Alf. 87.0 ft. Kk. 87 from Lima. Lycopericon

Gt (2) Semierect hent. vine on dry rodside. FIs. bright yellow.

Peku: Repto. Junin. Aurnrsyo Bought in Estanca de Cora. 28 dec 74 . 4626. Erythroxylum Coea Ecoal "gringuital From Huenuco. "Best chew" Price: 60 soles/pound.

Ean 462\%. Euthroxylum coea "Cayco Verde" - 60 soles/pound

Ecow 4628. Erythroy lum coca "Cunes Vegro" - "uges Huanteado" 
GHt $\$ 634$

untir?

USM (2) (1) Prostrate herb on rocks with succulentridlus. Infls. red.

4636. Peperomia pucu-pucu

Git (1) (4) subfleshy hent arising from round rhizome (bulb), qmoing in rockuall. Spiter crean.

4636. Solanum

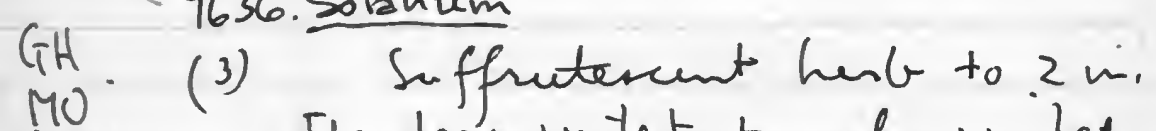
MIKM Fls.der viotet to pele violet. 4637. Calcedaria

GIt (I) Herb I m.tall on rad benk. Fls. lep yellow

GIt 4638.Calceolaria

(1) Jerb I 2 . tall on bank a long quebrada. Fls. bright yellow.

GH 4639 . Bomarea

(f) Vine in thichet along strean. Ovary ligut gree. Sepale dull red abrxially, creamy white adaxially:
with tiny merom spots at aper. Petals 
claved, abaxislly with ced clow $x$ midrib, bla de yellow green with spall mavoon dots near mangir; adaxialy claw crean, blade yellow gree with deune maroor spots throughout Authers grea,

Kin. Marher 176 , same localit. Ait. $11300 \mathrm{ft}$.

4640. Tecoma stans (L.) Juss. ex HBK. s.l,

(3) Trealet 2-3 $\mathrm{m}$. tall un roodside banks. forrotha deep ycllow.

4641. Cantua flexuosa $($ R. +P) Pers.

colt (2) det. $\sin 1979$.

Live: Shrub $1-2 \mathrm{~m}$. tall is thichet LyonArb. along strean. Caly $x$ green, corlla + Fila weuts creamy white.

(A)

ust (3) $\frac{\text { Ephedra }}{\text { Shenb with lax scandent }}$ GII $\checkmark$ branches, $1.5 \mathrm{~m}$. tah. Conee qreen. 
GH $\$ 643$. Solanum aff. lycioides L.

(2) Subshriet with sleuder ereet brancher. Cors la pale blue violet. Authers brighr orange. $\operatorname{det}$ A. Hungiher 1978.

PEru: Dept. H nanearelica. Near Husucavelica. Alt. c. $12500 \mathrm{ft}$.

Git 4644. Solznum nitidum R.tP.

sed Rozdsides. Slurub Im. tall. Ripe besries bolachist red.

PsRu: Dept. Husucavelica. $13 \mathrm{mi}$. Sw of Huancavelica, after Huama. A It. $13100 \mathrm{ft}$.

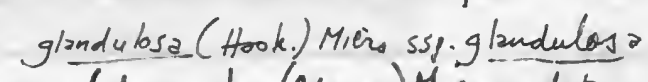
4645 Salpichroa of hirsuta (Megenthiens dest (8) (A) Semipristrate shrub giviving GH live against vertical rock faces. USM FIs. pendent; caly light green. col uev) Cordla light yellow. Frs. S 5 yellow green. Birm Mo (A) $\rightarrow K$ (A) $\rightarrow$ NY 
Prau: Dept. Huancavelica. Prov. Castrovirreyna. Anpacheta grande. AIT. $14800 \mathrm{fr}$.

4646. (Luasadeae Cajophora horrida Urb.t Gilg.

(4) USM Lerb growing in protection of unicating hairs. Petals red orange. Nectaries yellow Filaments white.

PrRu: Dept. Ayacucho. Ayacricho. I Jan 1975.

4647 . Solancum

Weedy herb zloug path in Ayacuchs. Fls. White.

4648. Esythrsxylum Coca

Leaves bought in Ayacucho market. I pand 50 soles. From "La Selva", Upper Apurimac. ('ween Ayna'.) 
Prau: Depto. Ayocucho.

Prov. Huamanga. Rosd from La Quinua to Abra Apacheta de Tambo. $3 \sin 75$.

Alt. 11500 fr.

Cilt 4649 .Scallonia myrtilloides L.f. var. myttilloides

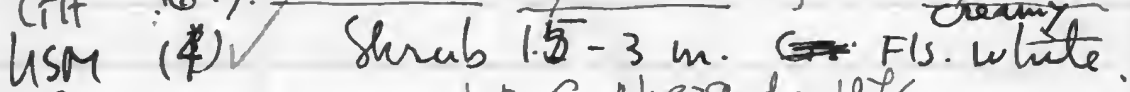
det. C. Niezgoda 1976.

4650. Bomarea

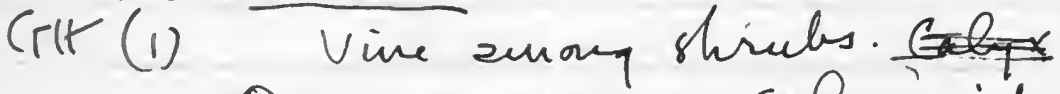
Ovary grey-gree. Calyx pinh. consu pullish yellow.

Alt. $12000 \mathrm{ft} \rightarrow$ Sarzech a spinosa DARreyt Sm . STracha punctata R.tP.]

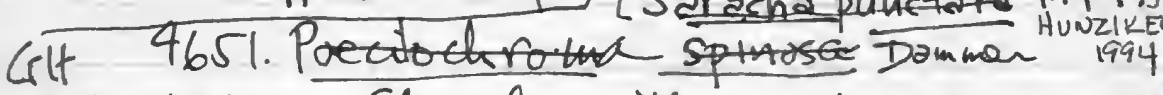
USM (5) Shrub with e ect stem t F ats 2 short horiz. branches. Crolla BMO + ive yellow at base, dach purple Col sudPTBOJbove. FR. black.

Prey: Depto. Ayzcuchs. Pror. Lu Man. Tambo, $A 1 t .104$ ro $\mathrm{fT}$.

4652. Clematis seemanii O. Ktz. usm (4) Voue on rozdside. Tepals Es cream white.

Grf $\rightarrow K$ det.C.Niezgoda 1976 . 
GIX $+65^{\circ}$

Ericas

usm (2) Shrub 1 m. o steop pe hillride. Pedicil,t corrola white aly reddish at bare, lokes lohite.

GH 4658. Baccharis sp.

Comp

UsM (2) Shrub $2 \mathrm{~m}$. Heade creamy White to pale pink.

det. E.zandini'ff

Glt 4659. Miconia sp. (Sect. (remenium) buelort. Shrub 1.5 in. in steep gpen Lillside. FRs. dark puple.
det Wurdack 1975

Git 4660, Cuplea

USH (2) Subshrub to In. Hypenth. dork red. Pefal red orange.

GH 4661 .

tembell.

(1) Tall herf $4 \mathrm{~m}$. in cloud foret. Fls. br. green.

GSH (2) L662. Lupinum 15 m. on hiluide.

Bamer blueviolet, punthite at middle. Lenigo blue violet, keel White. FKs, bmusisl blach. 
GH 4663. Peperomia

(1) Flech Lent on steep oper pad. band. Ifs. prich beneatl. Spikes red, fls green.

GH 4664. Demosthenesia pearcei (Brita)A.C.Smith Erican

(2) Shrubs to Im. on steep hillside. Cahx (t. gree. Corlla lt- fink

GIt 4665 . Piper

USOM (2) Shrub $3 \mathrm{~m}$. along quebrada at etge of forest spitid carb grey6 cown.

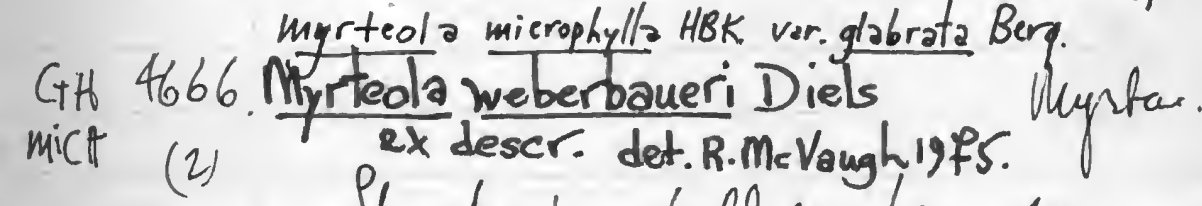

thrub im tall on shep sper hellide. Calyx pale grea. Betals pale pinh, resiniour taste.

USM (2) Begonia

Git (2) Kest on wet rad bank. Petioler teep pinhy under side of (f-it- pun cuary t abaxial suetc of sepalo deep priki adaxialy side t petals twhete. 
4668

Qnelied

(1) Epiphyte on treetrunh in

Ames wet forest. Lf. purple beneath. green above. Fis. dack wed with parred yellow sarkings-

GIt 4669. Peperomix

(1) Epiphyte on falle loq.

Semi-succulent. Spihe 1t. green.

4670.

Orchid

U. (2) Epiphote on tree trinhs in het forest. Fls. dull red purple.

Git 4671 .

(1) Kerb I m. tall. Fis. liqut grean.

46f2. Centropogon asteriscus (Winm.) Mevangh GIt seed-pros derbforet Lus. It green

USM (3) 26 ove ted purple keneath. s Outrspels qullowish to darh red purple. Crrlla greenoh crean. with purple hairs. Stame ctren palo gram, autler y darb furfe. Fi. It: gree.

det. S. Ippeser darb funcle 
Fit $\$ 6 f_{3}$. Fuchuis ovalis R. $+P$.

usy (3) Wert in lut forest $1.5 \mathrm{~m}$. Hypunth. $t$ sepalo pinh. petels deep pinh. Pedicel t ovary reddich green det. Kaw 1977 .

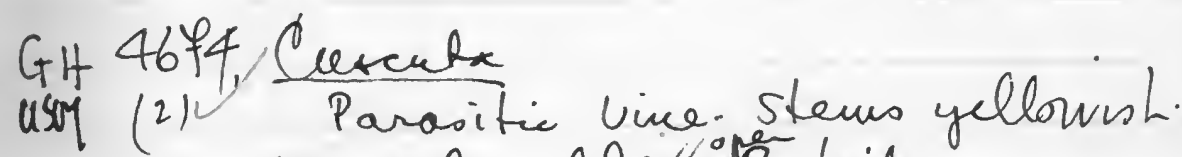
FIs. pale yellow! Rosdride

GB 4675 . Asplenim

(2) Fern on bench in wet for fern.

Git 4676 . Polypodium USM (2) Eiphyti fern a treetrmb r
Let forest.

Git 4677 . Asplenium

AB (2) Terer feic on bouk in wet foreot.

4678. Fuchsia denticulata R. $+P$.

sred Slirub $2 \mathrm{~m}$. Qu wet road

CHH (2) bank vean prol. coary yellow gree. Hy inth + seagal pint, sepals tipied grea. Petal deep

purh. Frs pole grea.
detrecol1977. 
CTH 4679 . Ranunculus

miKH (3) Nerb. Petale usM cadmin yellow. Cpen radiider - is wet places.

CH 4680 . Asplenim

USM (2) Herb on back in wet foreet.

Cist 468h. Asplenium

ISM (2) Ters. herb in met forest.

4682. Bomenea purplist green. Sepal ubaxirly orenge, adoxisle dep yellow, green át tip with purle spots. Petals: claw deep yellm, llade green with purle spot's.

GH 4683, Bomares

(1) Uive in thicket FRs. danb grear. 
Git $468 \%$

Usi (2) Cenptore ferm in wet forest.

Glt 4685. Bomarea

Oine in thichet. Ovary purplish. Sefols fale red-range, green at tip. Petals: daw yellow, pade gree, with purfle broun spots on adaxially surfore.

.

Rosd from El Tembo to Ayua. Alt. $7800 \mathrm{fr}$.

GA 4686. Ruyschio pavoniig. Don Margtaw usm (4) shrub on step roch cliff. F Fks. It-green. Nectary yellavgreen de Wet forest. $19 f_{6}$.

Git 4687 . Begonid

USM (2) plest (m. is cuet forest. Percaith pinkirl white.

4688. Corynaea sprucei Eichl. Balowph.

(I) (A) parphten in thich leaf mold,

GH ariaing from lange wordy thallur 6 in. dian. Peduncle brown Sufl dull solmon pink, 
Get 4689 . Clusia?

USM (2) The $5 \mathrm{~m}$. in wet fore 's Ny atoug quebrada. Fls. pale yel low is to cream.

Git 4690. Tradescantia?

4. (2) creeping henb at edge of forect. Fls. behite.

Git 4691. Pilea

Tertica.

u. (2) hesb $1.5 \mathrm{~m}$. in net forest. Fis. crean.

G14 4692 Trichantha sp-nor. GenneriosSelly (2) Shrub $2-3 \mathrm{~m}$. in wet foret. Peduit t colyx Gream
yellov. Corrutabull ned, vokes yellow.

GIt 4693 .

Uitar. 5 (2) in wiüe $4-5 \mathrm{~m}$. Buds green.

4694. Reperonid

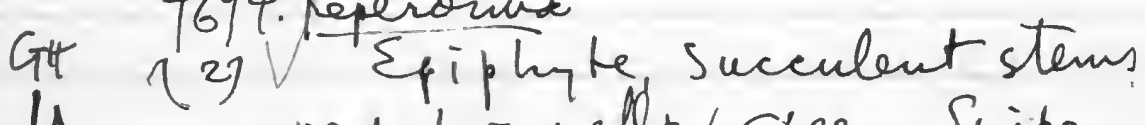
U. red. Lus yellow green. Ficks + fis. dark red. 1 
GH 4695 . Lycianther

BIRM (2) weah shut $1.5 \mathrm{~m}$ - Fls. light green.

4696 . Croton

uSM (2) Shrub $1.0 \mathrm{~m}$. Fls. Crear.

Fres. lequt bromist green.

GH $469^{\circ 7}$. Solzunm

BIRM (2) Treelet $3 \mathrm{~m}$. F/s. White. Frs. orange at maturity. Le '2' fst.

Git 4698 - Pilea

LCrtia.

u. (2) Werb im. Fis. pale grem. du forest slong quebrada.

olt 4699 ,

Cecurbit

USM

(3)

Keb. vime 4-5 m. long in forest margin. Ovany t hy pauthir pale gree. Corolla yellow-orange.

GH $4 \varphi_{\infty}$. foret. FRs. dark given mottled Wwith baikt green spots. 
USM 4fo1. Efidendrm

crop in wet forest, $3 \mathrm{~m}$. Tall.

Rachis t rvary dark broun. Bract t sepals reddigh to purpleit binni, repalo dull yellow adexiah. Croll dar ted-purple. Lif cream.

aris $4 \varphi_{02}$.

Crchid.

(b) Epiphigte on tree trunh i moro foret. Sepals violet. Petale white with vijlet tinge.

4603.

Avalia.

USH

(4)

Uubranched tree $4 \mathrm{~m}$.

US tall. Buls legut green. Lus. rusty brown keneath.

4704 Dioscorea

USM (2) Vive $2 \mathrm{~m}$. Pls - dull yellow. Forest margin aloug trairl 
Rod fim Tambo to Ayma. Alt. $5500 \mathrm{ft} .3 \mathrm{dan} 1975$.

us $4 f_{\text {ros }}$ Begonia parviflors

Gt (2) Unbranched herb $6 \mathrm{~m}$. Lf. darb red keneatly green zbove. Frs. brown. Fst. Margin suevary.

Git 1706 . Solzunum

BIRM (3) crusetshrub $2 \mathrm{~m}$. on open roadMo Side Lfs. danh gren above, whidisf fencath. Fls. white. Frs. dashgreen.

Alt. $6100 \mathrm{ft}$.

Git 4fo f. Solanum (Lycianthea) no Shrub $3 \mathrm{~m}$. on ope rosdide. BIRT Covrlla deep violet, feretim pale vislat.

Ifos Heliconín Subulata R: + P.

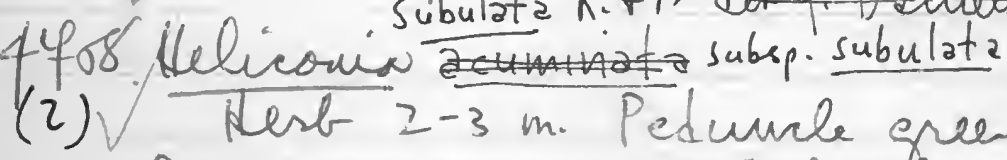
Bracts t vachis red. Ped cib red U. Gvary grea. Crolla yellow. Frs. GH blach. Friest marfin. det. Andersson 1984. 
A $1+.47 \% 0 \mathrm{ft}$.

Gt 4709 . Heliconia lingulata R. P P

GI (3) Nerb.2ma or quen rosdide USM gat edse of huside. Raslis,

seed PTBCS brasts, ped ice, bracteale, ovany bright yellow. Fres. black. det Danils Hit. $3200 \mathrm{ft}$.

4709 a costus

GH $(-4+\infty)$ Nerb in thichet alang 4 (3) rod, $4 \mathrm{~m}$, tall. Bracts liqut usy qreen apicaly red below overy White. Calyx red, Corolla deep range - red follominarde.

4710 . Calather GH (A) ${ }^{3}$. Herb $2 \mathrm{~m}$. in thichet along Bracterles Trlite to crea. sepel pale greacion white. Crolla tubet petilo crean. Staminode It. brows. Stamera darb bosen. 
Peke: Depto. Ayacucho. Prov. La Mar. Ri s Apurimac. La Mejorada. $1920 \mathrm{ft}$. alt. Between San Francisco and Sente Rosa, Dist. Caprice.. 4 Jan 1975 .

fir. Erythroxylum coca

soil (3)

(A)

Seed to Duke 5 hectares planted in rows. cs. Soured f con seed. Plants / wive 10-15 ECoN years. Harvest every 3 months.

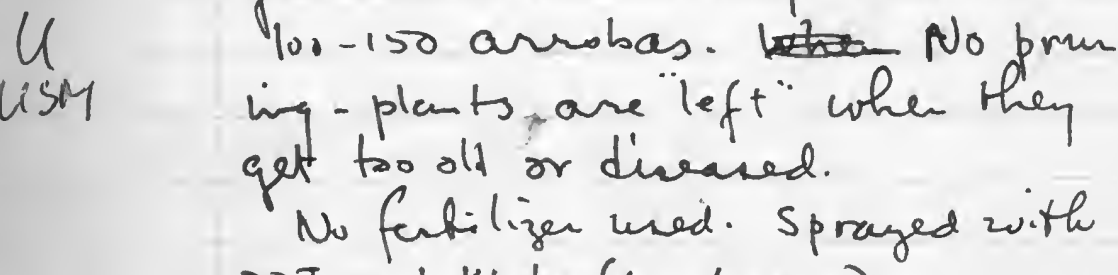
DDT and Kin be (banbasco).

Planted $1-3$ licker apart, rows $1 \frac{k}{2}-2 \mathrm{ft}$. z pant. Soil red. vertical furrows $3 \mathrm{in}$. deep. 1. toknay - trunk green burned 2.1 ausajelma $\}$ to make tara. witches broom- atop lichens-? enfermotad Price: 1000 sole /arroba. Tax in Machenta \$55:20/arroba.

Shrub $1-1.5 \mathrm{~m}$, truck to $2 \mathrm{~cm}, \mathrm{gre}$. picked I wreak earlier. Les. yellowish gree, pale gree beneath with 2 distinct paler lines. Pedicel t calpe pe goer. Petals cream, becoming yellowish. Frs. dull yellow, becoming duel orange, then red.
Ils. lImy Short styled. 
4712. Enythroxy/ur cora

(a)

usM Plantation on hillside, deck Foin 2 boom sol.

Col Shrob.5-1.0 m. tall hus. dah US green above, pale green beneath

Ecol some plants chlorstic with $K$ light yellow green leaves. Soil dark

(A) $\rightarrow K$

4713. Engthroxylum cora

$F(3)$

Git ned older planting fuel if weeds US 2 and witches brown and lichens.

\begin{tabular}{ll}
4731. & lichens \\
& on 4713 ! \\
\hline
\end{tabular}


Spilanthes a lba L'Hen det. H. Robinson

1979.

4714. Spilanthes acumella $L$.

Coluy.

ECoN (2) "Kubikiro"- heads used

US for trothoche. Anaeathetic effect.

Wead in coul. Heads pale yellow.

$\operatorname{det} C$ raci $q$ reene 1975

4715. Commelina longicaulis Jacg. (1) Hesb in cozal Fls. bright blue.

Ecol 4716. Amaranthes dubius Mart. ex Thell.

(1) weed in cocel. FIs greenish white.

4717. Jussiena erecta $L$.

USח (2) West in cocal, I m. Petals goldew yellow, ovary dark reed.

ECoN 4718 . Eleusine indica (L.) goerth. gram.

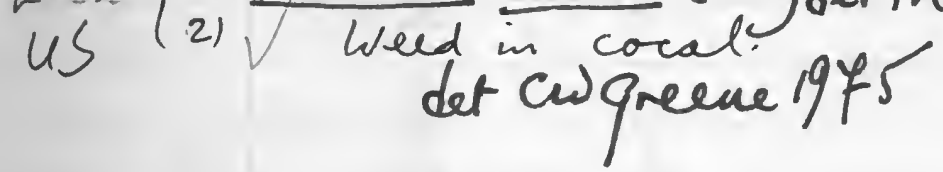

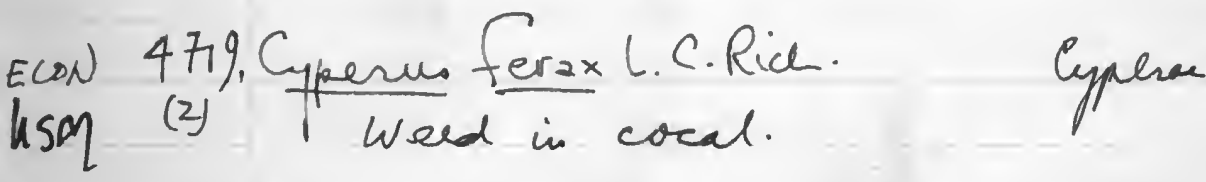

4720. Ageratum conyzoides L.

USM (2) weed in cocal. Fls. It. blue. det Tp. 1975. 
Us 4721, Setaria geniculata (Lam.) Beaur.fram ¿coN (2) wead í coral. 75 .

US 4722 . Paspalum conjugatum Berg. gramin. (2) det cu qreeue

EcoN 4723 . Panicum laxum Sw. gram.

det. Craig Greene'to

ECON 4724 . Vernowia?

Conep.

us plesto Slerub $2 \mathrm{~m}$. Sterile. Rosdride. Stem \& nots used to make "tresa" of. Plowmen 7939. Toera sample.

GH 4725. Dimesscostus argenteus (R.tP.) Maas U. USM. (4)

F foro

West $4-5 \mathrm{~m}$. in low lying suacupy place oloug road. Lus. silceris pulescent beneath. Broits grelling

appendage green. Broctealert callyx pole tollowish Labellim (Staminode) trandracent pale ereaning with seed PTBG go den yellow hairs on adzoid imbsurfoce. 
Stromen creann white, limb extension $1 t$. yeelow. Lufl. $44 \mathrm{cen}$. long.

4726. Lonchocarpuis urucu Killip + Shaw $\varepsilon_{c o}$ (1) "Kube" "barbaseo" iscandent slerub $2 \mathrm{~m}$. tall cult. for roots. Sterile.

Roots dried, shipped to Lima to extract rotenone. det cugreane 1975

4727. Bomarea

(2) Vine in thichets, frequent. sopals orange. Petals yelloworsuge.

4728. Bideus pilosa L. Var. miñor (BL) Scherf Gron (1) weed in cocal Rays whiteDisc yellow. det. C.qreen fo. Above Sen Francisco.c. 3300 fto 4729. Erythroxylum cora Laun.

ECoN (2) shrub $1.5-2 \mathrm{~m}$. in old planting:

MO (A) Interp
yuca?
$\rightarrow \rightarrow$ 
Machente, between Anna it San Francisco. 5 JAN $1975.2^{\circ}$ Thickets. AIr.?

4730. Acalypha

Git (I) Shrub $2 \mathrm{~m}$. on hilt behind

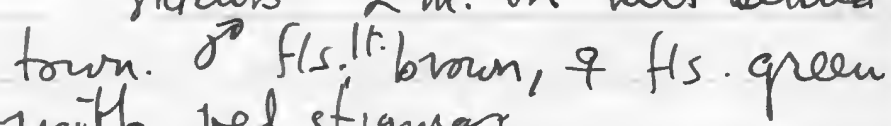
with red stigmas

4731 . Piker elongotum Val

Git (2) "latino" deteR. Colleges 1982. Shrub 2 m. tall behind torn on hill. stems + petiole red. Spikes cream.

4432 . Curcuma

u. (2) palilto" (turmeric) Jingit Herb to I m. on hillside behind town. Bracts pale greenish white, cream tow and apex of spice. 'Fils. yellow. 
Prese: Depto. Ayacucho. Prov, La Mar. Between El Sambo and Agra. Masinga.

Dry thorn screech. Alt. $10500 \mathrm{ft} .5 \mathrm{~J}$ an 75 .

Capphora of. Cirsiifolis Pres

GII 4733. Lass ace cajophora pierce

USOY (2) Low growing herbaceous vine.

Petals red-orange.

L. Set.

GIt 4734. Solanum lycioides. Aturizeer 1976 Birr (3) Densely branched, armed shrub Mo $1.2 \mathrm{~m}$. tall. Conlla white to pale violet, anthers red-orange.

(A) $\mathrm{K}$

USM (2) Twining vire in thickets.

Git Ovary greegrees. Sepals abaxial dull pint to bright pint pale green near apex, warily whit id pinch. Petals clawed: claw Cream to yellow with deep pint medial strife. Blade bright yellowish green with desk brown spots on ada rial si face.

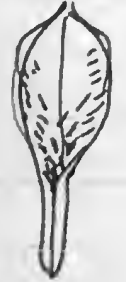



$\frac{\text { DUNAUA OBOUATA (R.AP.) DAMMER }}{\text { S.D.SMITI (WIS) } 2003}$ Dunalia spinosa

GIt (5) (A) Arued shrub 2-3 m. tall, usM seed in thichets almg radsides. Corlla $M 0$ ind deep blue-violet. FR. yellow at BIKM 2 maturity. Frequent.

(A) $\rightarrow K$

Iricho-

$4 f_{3} \%$ Cerens $p$.

Usy (2) Cactur $3-4 \mathrm{~m}$. tall, branched c*t forming clump. Syparthin yellow green with dach 6 mox hairs. Outer tepale pale grea near baxe, dank maroven u upfer half. Inver tepal pale maroon to creany white. Filsments $t$ Style very pale grea, stigmas yellonish. det. m. Flores 1977 .

$$
4737 A \cdot \frac{\text { P2sifiona }}{\text { seed on } y \text { - }}
$$


Prru: Depto. Cuzco. Prov. Cuzco. Cuzco. Ruinas de Sacsahuaman. Alt. 12 Jan 1975.

4738. Spirzenthes (live) Wert on steep earth bauks among
only

4739. Solpichroa

Git (1) subshueb $30 \mathrm{~cm}$. growing in rock crevice a mong ruins. Fls. greanis. yellowr.

PERu: Depto. Cuzoo. Prov. Urubamba. O.llantaytambo. A 1 t. $9200 \mathrm{fT}$. if $\operatorname{con} 1975$.

4740

Orchid.

AMES (b) Terrest. henb on rocks forming clumps. Fis. pale browsish purpit.

Hit. $10000 \mathrm{ft}$.

Git 4741 . Lycopodium

(1) Heif in roch crevice

Alt $q 000 \mathrm{ft}$.

4742. Dioscorea.

Gt il Ving shronq vocks. Fis. greenial. 
Alt. $9500 \mathrm{ft}$.

4743 . Mutisia acuminata var

Crmpopite

Mutisial

(4) condolleana (gordn.) Cabr.

Cuzeo toad north if $Q$ llantaytanbo.

Git lufl. purplish. Rays egellow Us orange. Styles red orange.

Psku: Depto. Cuzcs. Prov. Urubamba Road Olintay tamba to Chacelloy. 17 Jan 1975. Alt. $10500 \mathrm{ft}$.

4744. Tropaeolum Crehabambas Buch. GH (4) vine on open radbanh. F Cugo clyx It. red, sper deep red rauge. Petali gorder yellow.

4745 - Bomarea

GH (2) Vuñe in thichet. foto Sepal derp red absicill, UsM dull yellow adaxially. Petald' yellow orauge with red spen and costa

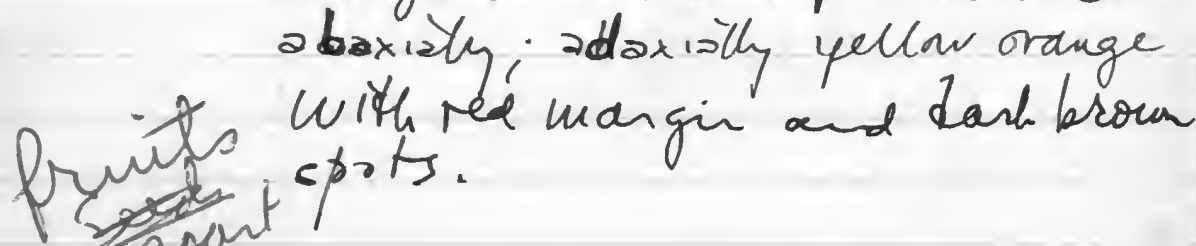


PEru: Depto. Cuzeo. Prvv. La Convencion. Alt. $11500 \mathrm{fJ}$. Kia. 144 fion Cazeo.

GH $474 \%$ Salpichroa didierian Jaubut To 8$)^{3}$ Vine climbing on treetrunk, ro foto $5 \mathrm{~m}$. hiqh. Caly $x$ peen with us curco brownicl lobes. Corrlla yellow; USM pendout flot.

(A) $\Rightarrow M$

Centropogon isabellinus Wimm.

4747 Sipho canpy det. B. Stein 1985. Componuml.

GH folt (2) Hent on rood sidebacks.

$k$ Celyx purplish green. Conda lip dorsach pinit, ventrally pale sead PTBS green, cyper lobes pinh. Stamen tabe It-green.

4748-Solenum

GH (3) Shrub $3 \mathrm{~m}$. tall. Cosslla white. Cues Fr. green. BIRT

4749. Fuchsia denticulata R. AP

GH radbanks. Hypenthim, sepals it K detala red-orange. det. Ken 9977 . 
Ait. $9760 \mathrm{ft}$.

CHH 4750. Colceslaria zanatilla Kränzlin

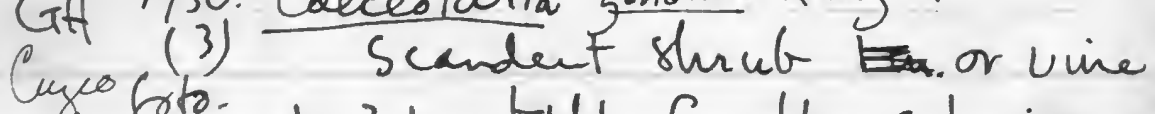
K foto-1-2m. tall. Corolla cadmin yellow. det. U.Molau 1982.

A (t. $10700 \mathrm{ft}$. (HBK.) Endl.

GH 4751. Colignonia parviflora Nyctag.

U (3) Shrub 1-2 m-tall stong quemada. I or more uth

Ivs. white Fls-puih 197

4752. Tropacolu coinebambe Buch.

GH Foto. Extensive hent. vine on steep Cupoo open baut. Calyx, spur +2 lower $S$ seddor petals bright red-orange, upper

(4) $) K$

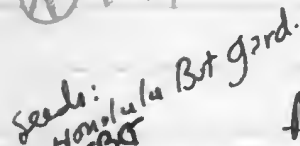

Alt. $8300 \mathrm{ft}$. Abare Alpamayo.

4753. Conno iridiflora R.tP.

(B) (13)

fors. Hesb $4 \mathrm{~cm}$. tall is clumps Auzes along rodsidesongents $U$ USM and along quelugdas. Bracti U. ovary + rachis dach purfle. GH Cork Co purple. Staminode teep is 9 canmine red. FBs. yellow green 
Alt.8100 ft. $17 \operatorname{san} 75$

4754. Camna leucocerpa Bouché = Cjoegerianus

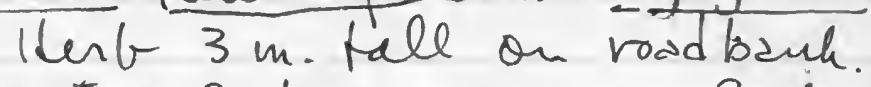
Bract pale bisusist gree. Rackis U.- I) pale burum. Ovary green. Celyt reddich dree Corlla prele oraye. 1t. green with ted ispines.

A (t. $5800 \mathrm{ft}$.

4755. Revealmia thyrsoidea (R.+P.) P.tE. fis). 1derb on steph hallside in cuefo shade' $3 \mathrm{~m}$. tall. Rachin It. gree brown, becoming young frut resdish Remais of caly grange.

Alt. 5 f zo ft. Huayopata.

4756. Erythroxylum cora

Shumb $1.5 \mathrm{~m}$. tall in tea plan.

fors. tation, interplanted with coffec, ECon front trese etc. Lus. bright greeabove, culli pale gree beneath with 2 destinct lines. Fres. orange to red-range. 
Econ) $475 \%$-Camellia sinemis.

I (2) Extcurivel cult. Shrub 1 m. tall. Corslla white. Frs. green.

Peru: Dept. Cuzes. Prov. La Convencion Alt. $3500 \mathrm{ft}$. Maranura. Haciénda Suandor. Isidro Sayre Blancol

UST 4758. Euthroxylum cora

(5) $\mathrm{V}$

Exterioive coca plantations $F \quad 3$ year old plentri pruned back to ground every Unprmed $1.5 \mathrm{~m}$. tall, pruned do. 5-1m. Young fruitigreen.

4759. Copsicum baccatum L.

Eron (2) subshrub $1.5 \mathrm{~m}$ in coea RNG field. Petals white. Frs. still green

$$
\operatorname{det} \beta \text {. Pichengill } 1975
$$

N. 
4760. Couma glauca

(4) (3) Herb 1.5 un tall in suasup Cuzuoplace aloug road. Bracts pasee. U. Rachis glampoas. Ovary th green. Git Calyx palegrea. Con tha pafe ycllow. Stzuninode yellaw. Stzuner yella.

P乏Ru: Prov. La Convencion. Depto.Cuzco Quillabamba. $18 \mathrm{Jan} \mathrm{f}_{5}$.

Alt. 3400 ft.

4761. Solanum Caricaefolium Rusby

GIt (2) Surmb $1.5 \mathrm{~m}$. tall along roadside BHFF $K$ in Quillabemba. Fls. whte. seed PTBG FRS. orange. det A. Child RTF

Road to valle de Santana Pôtrero.

4762. Pogonopus tubulosus (DC.) Seem. Git (2) Scandent shrub $2.5 \mathrm{~m}$. Practs $K$ deep car unino pinh, Cor lla dull pinkto grepple, lober cream adaxially. Alt. $3500 \mathrm{ft}$. det Kew 1977 . 
Git 4763 Alt. $4800 \mathrm{fT}$

BIRM (3) Rosdside shrub 1-15m. tall. MO Corrlla fleshy, white. Frs.green.

$$
\text { Alt. } 4780 \mathrm{ft} \text {. }
$$

4764. Solncem of. 2ff. S. Torrum Sw.

LSM (3) Rosdside Ghuib $2 \mathrm{~m}$. Tall. GH LF. underside white, dark shimy sed Prot. green above. Thes Crea

4765 . Inge

(3)

USM Toe with spreading Crown. Ny not blanted but uned to shade

Alt. $4800 \mathrm{ft}$.

4766. Costus off guanaiensis Rusby

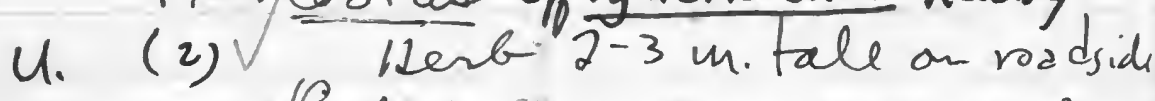
Git thicket. Bracts red, exposed arpendage it. green. Ovary white. Calyx red. Cor Cla pale cram-ylla stiminod. 2baxially creany yollow, adasiall pinchrouge. Itzmen abzxizle creany yellm, adoxislly puil: 
A It. $5400 \mathrm{fr}$.

4764 Erythroxylum raimandii $0.50 h u l 3$

(8) (A) "cora coca.

fats Shrub $1 \mathrm{~m}$. to treble $4-5 \mathrm{~m}$. tall euro mostly with several trunks ca. $5 \mathrm{~cm}$. USM diameter, representing stem sprouts u from older trees.

us Growing in open pasture with habitat. Git... Oidium on hillside beside extensive Econ coca plantation..

CoL Stems reddish to greyish brows with It. bour lenticel. Stipule persistent, Woo. pole, yellowish green be coning brown. Aw M I is, dark green a above, dell or somewhat Un Shim pale green midrib and int veii ins; beneath pale dull green, with slight h poles midrib and nerves, the veinlet appearing darker queen, finely reticulate. No flowers or fruits. Flowering" in March. Many plants virus in fected and/ur with l witches broom.

Leaves of this Coca are meed to adulterate commercial coca by adding $4.5 \mathrm{lbs}$ of E. tainendi per arroba. Leaves said to "picks" the tongue. 
Alt. $3600 \mathrm{ft}$.

4768. Euthroylum rainandii

4SM (3) "coca coca"

ECON Scandent shrub on Steep bonks, us $1-2 \mathrm{~m}$. tall. Sterile.

Alt. $4600 \mathrm{ft} .19 \operatorname{Jan} 1975$ Econ 4769 . Erythroxylum coca Lam.

US (2) "Colt. Shrub $1 \mathrm{~m}$. tall partial Cult. Shrub $1 \mathrm{~m}$. tall $\mathrm{m}_{1}$ sum s long rood. Frs. red. growing interplanted with cafe, Cobcasia, citrus.

4770. Enthroxylum coca Lam.

- $\cos (2)$ us

Cult: Shrub $1.5 \mathrm{~m}$. tall growing shade binder coffee. Sterile.

4771. Erythroxylum cons Lam us (2) Belt. shrub $1.5 \mathrm{~m}$. tall ginning in shade of coffee. Sterile.

4772 Ecopthroxyl cum coca
$1.0 \mathrm{~m}$. Us Cult. Shrub along rad in partial
sum. Frs. pole green. 
4773. Enythorylum caca

acon (2)

Calt. shrub $1.5 \mathrm{~m}$. tall. Sterile. tu shade.

A It. $4400 \mathrm{ff} .(1333 \mathrm{~m})$.

4774. Enthroxylum Coea

$\cos (2)$

us

Cult - shub in partial shade, $2 \mathrm{~m}$. tall. Fr. brifht red. On coffee plentation.

$$
\text { Alt. } 4780 \mathrm{ft} .(1448 \mathrm{~m})
$$

4775. Erythroxylum cora

Seed (2)

ganders Cult. shrub I m. tall planted in oluke verticar rows of steep hillside, woeds E(i) prevelent. Receutly piched. New hs leaves pale yellow-green (chlorotic?) turning bown in press overnight (ef. E. raimondii). Heks. abundant, small, pale yellow green. 
4776. Erythroxylum coca

ECON

US

Shrub $1 \mathrm{~m}$. tall in fall sum on open hillside new vellow-green. - Frs. afyundant, green then yellow. orange, then briqht red.

$3600 \mathrm{ft}$. Alt.

4777 . Erythroxylum simondii

Cunco

usM Shrub $2.5 \mathrm{~m}$. tall zlong

(8) rosd near Cumenterio de Quilla-

ECoN bsmba. Lus. dull to somewhat

Git shing above, pale gree benecth s witt dart gr verilets. no linea.

Mo Greolar tegion scancely distin-

$K$ Guishable. Phs light yellow-gree us huith dant pumple pequentation,

Very frequent. 
Peru: Cuzco: La Convencion 4778. Bomarea

U. (2) Uine in thicket Ovary pumpleGit black. Syals pale prih. Petals crean with purple brown spits.

4779. Arrabidaex sello i (Sprem.) Sandw. (2) viue in thichets. Cordla pinh-violet
det.A.qentry 75

4750 . mesechites.

Apocynac.

USM (2) Vive with milky sap. Fres. green Git turving boson.

4781. Duranta sprucei Briq.

NY (2) seandent shrub!. Fis. pale violet. Fins. orange. 
lima

- palta haranj

- cofe

pina

- 2020

- Inqe spp.

- bonara

Coloessia

fripl

- mri3

cuturbita maximo papaya achiote

Caña

canotes

cabrya

kigan tea's

yuca

Annona

malg?

Arracacha

lechuga)

mani

col

rocoto

ruda

higher

celsutro

pot hear?

lupin

Nunthosoma sp.

Luсnuma? 
Precu: Depto. Cuzco. Prov. La Con vencion. Quillabamba. Valle de Santa Ana. 20 gan 75

A it. $4200 \mathrm{fr}$.

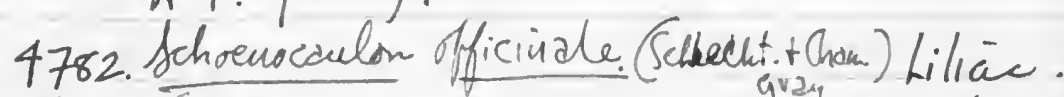

$F$ (4) Bulbif hent on steep banks. - Cuzio Spibes pale green. Fis. creany

Grts white, foetid.

det. T.Plowman 1978

4783. Podandrogyne

Capparac

shrub $2.5 \mathrm{~m}$. tall in shade

(3) at extge 1 quebrada. Fis. red oraye

GH U Rachis t petioles red-pinple.

4784 Physalin

Glt (2) kerb $1.5 \mathrm{~m}$. in shade aloug BIRY quebrada. Corrll pele yellow, darh 6 onon near base.

Ny 4785 . Inga

Tree $5 \mathrm{~m}$. with soreading Chow Calt. for shade? Fildancuts white.

4786. Copsicun baccatumL.

ECON (1) Shrub $\frac{1}{1.0 \mathrm{~m}}$. Frs. peleyellors Along trail on hillido. dét Barbara Picheragil 1975 
4787. Citrus

$E \cos$ (2) "liven duce.

Cult. tree $4 \mathrm{~m}$. tall. Petals white. Firs green, yellow at maturity. Flesh' pali greenish

4788-Capsicum baccatum L.

Econ (i) Herb 2longtrail. Corolla set B. iv pale cream- - white with yellow pidengils spots with in at the lobes. FRs. piderits yellow-green, very pungent.

$u$ 4789. Dichorisandra

(3) Scandent herb, on vine, USM

$\checkmark$ hanging firm rod bank. Calyx pal blue. Corolla deep blue-viotet. Fra yellow greer on lower half, deep wine ted on uppers

ECoN (2) 479\%. Citrus

(2) manderima

Cultitree $3 \mathrm{~m}$. Hall. Flo white fragrant. Fr. green, flesh orange- 
Alt. $5800 \mathrm{ft}$. "Seemannia sylvatica 4791. Gloxinia syluatica (HBk) Wiehlar Geruh.

(1) Terb on open hillside chumg qrasses. Sterus red. Pedicèl ted. Colyx pale green to reddich. Corlla red-orgugé detuíflated.

foriss 4792.

Qrahid.

u.

(2)

Tuberieled orchid anong grasces or steep hillsider. Calx pich. Petals white bzsally, psle pinh towand tip. hip white, middlehse. deep margin.

4793 Hesperoxiphion peruvianum Baker Ir dac.

a. (2) Bubiferour herb w ore hillute Git bulb! among grasises. Fis golden yellow.

4794. Dalechampia

UsT

vine in open thichets on steephillside Bracts white tinged fainth with pink. Glands pole green. eyathea red.

4795 - lavija of pungens (willd.) Radlk.

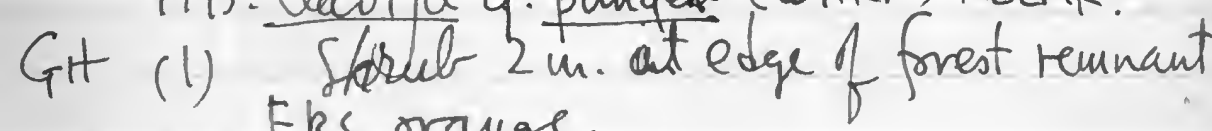
Fks. orange. 
4796. Diehorisandra

U. (3) litert-1-1.5 m. tall in cutover forest remnant. Calyx pal green. Corrlla it to deep blueviolet.

Ames (1). Terrestrial orchid in deep shade in forest on steep hillside. Fls. light creen througlout.

GIt 4798, Peperomin

(1) Succulent \&iphytic herb ow trectrunh. Stew red. Sphes yellow-greer.

Glif 4799. Diastems sp. gencen.

(2) keb in moist forest margi on hid tidg ienter 75 scarelet.

GTH 4800 .

Cusco(3) Epiphiptic fernontretruak.

Git 4801 .

Brermannic

(A) Freoly Sapsophygte in humus.

(1) Steun aed. Infl. + flowers white. 
4802. Slophoglosou

(2) Cespitsseferm in forest on steep hillside.

4803.

Crucifer.

(2) Hent in moist hillside forest. Fls. creany white.

$480 \%$ Centroprgon
Git (I) Herb to $0.5 \mathrm{~m}$. in chacarar recently cut. Corolla deep carnine pink.

Git 4805. Piper

Cuifu (3) Slumb $2-2.5 \mathrm{~m}$. cir usodo on steep hillside.

Git 4866 . Solanum

(1) Kent $0.5 \mathrm{~m}$. tall li cut over forest. Retals greemis, reflexed Stamens ducl yellor. Frs. Wrinkled, blackijh-green.

4807. Lycionthes
Git (2) $1-5 \mathrm{~m}$. Corolla brounish BIRY purple. 
Thalictrein

Gik

4808.

4SM (2) Hent 1-2un.tall m oper dearing in hillside forest. Fls greeuish.

4809. Sepsicaun Pubescens R. $+P$.
usm- (6) Cult? het in Chacara in RNG tors steep hills side foreot: $1 \mathrm{~m}$. Foll. ECON (A) Cordlla deep purfle.

F 4810 . Podocarpus harmosianus Pilgar Conifer.

(3) (11.) Tree 6 m. tall on hillide lisis dictarked forest. Sterils. Only list ow seen.

4811. Peperomic

Git (1) Epiphite, Heahy in cutorer fore.t. Stewo red.spites pale qreevis - white.

4812.

Orclise

Ames (1) Terrest. in moist forest. - Scape reddish. Fis. pure culite.

4813

Orchis

Awes (1) Epiphite in cut over foret.
Flsdelieddion lown. 
det. Herkblots.

20 Jau 1975 var. estrellanus E. Wimm. $\mathrm{CH} 4814$-Centropogon urabambae E.Wimm. Cawpaun Dire hanging from treetops oloug trail. Crolla bripht carmine sed PTBCr pich. det C. Niezgoda 1976. live feruanly live. U. Ch. El: El. Al. Wamea 1781! Alt. $4800 \mathrm{ft}$.

4815 . Erfturaxymen coca Lam. Ecow (2) cchipe cona"? us Calt. Shrub $1 \mathrm{~m}$ in open killside. Lus. dark greer zbove, pale greer beulath.
Frs. gree.

4816 Erythroxylu coes Lam.

Seedlings Gnong meeds near Coca plentation. Lus. yellowish.

Econ 817 Erethroxylum cosa $2 \mathrm{~m}$.

us (2) Colt. Shruh Im in partial shade in coffee placeting. Lus. dark grew above, pale green beveath. Frs. green.

4818. Eruthroxylum coca Lam.

ECON (A)Fl's) Coll-shrub Im. tale in placutation FH (3) Elong trail. Lus. bright yellowish Greer, paler beneatt. FIs Creann-yellar. 
$16005 x$.

Alt. $5300 \mathrm{ft} .21 \operatorname{Jan} 1975$

4819 . Erphroxylum Core Lan.

(A) ff, "core"

Git (4) Shrub in. cult. in partial
us shade under coffee t In ga.
CoL Fils. Creon. Frs. olive green.

Alt. $4500 \mathrm{ft}$ - $1305 \mathrm{dil}$

4820. Erythroxylum Cora Lam.

uss (3) cult. Shrub I m. on steep ope Econ hillside. Steichen with zitis brooms.

3600 ft.

* 4821. Erathroxylum Cora Lam.

seed to

Punch (4774) Heterogeneorer plantation, ganders cree interplanted will yuma, cafe connote, activate, frijol, lain, shaded by toga. Rows 2 coo after vatu with rows If cafes, move C. I m. apart, horizontal

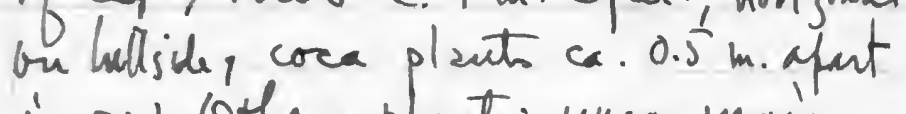
in sow. Others plant: ymca, main, frijol, mixed in more or less randomly. Cores planted with several (2-3) plat per "hill". When 3 years sld, cat bark to 
about 3 inches form ground no pruning of upper branches.

with erect braves.

4821 a shrub" $1.5 \mathrm{~m}$. tall. Firs. ovoid, us 2 dore deep yellow.

Econ $)^{d o r}$ b. Shrub l 1 m. Frs. Jlobrose-ovoid us bright red, shiny, when mature!

Econ 3 C. Shrub with spreading branches, Ivs. US small, dark green above, pale green beneath. Frenits long elliptisoid, light green.

Eco) d. Shrub $1 . \mathrm{m}$. tall, branches us dxpespreadivi Les. dank, legit gree above (young), pale green beneath. Frs. fusiform $)$, (t. green.

usm - e. Shrub $1.8 \mathrm{~m}$. FRS: oliver green, Econ dore to duel yellow to rage. vo dark green, shiny firm membranecoos, rubbery to touch.

$\Leftrightarrow K$ 
Weeds in and a mund cra fields. 3600 ft. 4822. Eleusinuindice (L.)gaertu. Graminew ECoN (1) Spihes It. green.

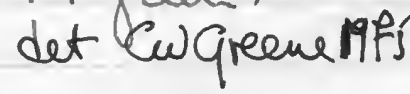

EcoN 4823. Molvastrun cor omandelizinum

(1) Hert. Fls. yellow orange. (L.) gorche det CWG eenets

4824. Emphorbia heterophylla L.

Exal (1) Hero with white leterx Ovany t glando bri green. Petiole red. Fls- Lull yellow. det Culgreene 75

4825. Hyptis mutabilis (Rich.)Briq. Lobrat. Usm, (2) stherb $1-1.5 \mathrm{~m}$. Strondy aromatic. Econ Fls. lavenderpinh. det cusqreene 4826.

Gitt (1) Tessootr. Fera on burren billsides under coffee.

Econ (1) $\frac{4827 \text { Phyllanthus orbiculatus Rich. }}{\text { terb on vadside. Les. Canh }}$ det. qpeen-purple above, dull greyligiss purfe beneath, Fis. dull puiv. Ecan 4828. Conyza bonariens is (L.) coonq Comst (i) Rest. Capitula creany yehor. 


$$
2 \cdot \tan 75
$$

4829. Porophyllum ruderale (face. Cass. Couprosit. EloN (I) HerG. Invilucre qreanito purple Fis.purplish. det Cugreene 1975

4830. Desmodium

(1) Stichy herb. Fres. green.

483 1. Solanum nodiflorum Jzea.

Esm (3) Herb under Cffer-shade. Comla BIRM cohite. Fres. dail oreen tirning black. ECoN 4832 Erivehloa punctata (L.) Desv. Gromimes (1) Hent on Slope. det Cugreene

Ecad 4833. Amarauthus hydridus $L$.

(1) Stems red Braits It.green.

Econ t834. Euphorhia hirta L.

(1) Stact herb. Stews t infl. dull red. det $C u$ greene 75

Econ 4835. Cyperus cayennensis (am.) Britton Sedge
BH (2) Oult hillside, shade. det Cis greecu f?

ECoN 4836 . Cenchrens viridis Spreng.

(1) Roadside. Fks. light green with red spmils.

$\operatorname{det}$ (.W. greene 1975 
(Raddi) Nees

4834 Trichachne sacchariflora gramin.

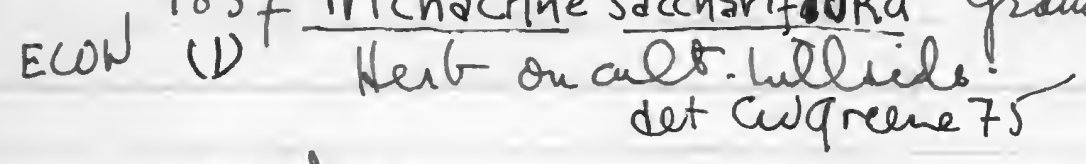

4838 . Juga

USM
Ny
Git

(1) Tre 4min in coffee plsutation. Colyx yellow greer. Fonda + filament colite.

4839. Lautana armata Schau.

NY (2) terb I ve Corclas dep pinh GH in center of head, gellow orauge at peripher moldeuke 1975.

NY 4840. Lautana maxima Hayek

GH (2) Wer Im. Bracts grew. Conllag levenden pink.

NY 4841:Lantana armata Schau

GH (2) Hert $1.5 \mathrm{~m}$. Corllla tube dep red orange, limb red-orauge to yellor orange. det Moldenke 1975. 
La Trua. $3600 \mathrm{fx}$.

4842. Lautana canescens H.B.K.

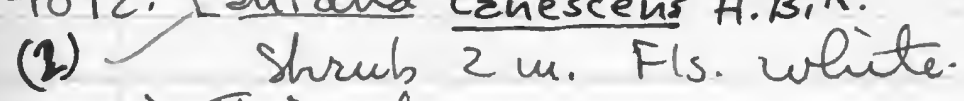

in Thichets.

det. Moldenke 1975

4843. Talinum paniculatum (Jaci) gaertn.

Git (1) Fleshy herbon rocdside. Fls. yellow!

4844. Erythroxylum rzimandii 0.Efchoolz

GIt (3) Toee 5 un.tall on edge of

ECQN w oliff in full sum. Lus. dank green Shing, above, pele green bemeath, call yellow green to red range.

Quillabaub. Garden of Hotel Comercio. 4845. Calathea Pavonii Koern.

(2) Hent. Lus green a bove, pale

A-IP (A) $\rightarrow F$ grae below with very fint redil bunds. Bract, green. Fis. yellow.

Tavier Sernaque-foto of kids cosreo Ceitral Quillabamba 
PEres: nept. Cuzes. Prov. La Gonvencion. Vegr Elagrate on road to Johusyacu. $24 \mathrm{tan} 7 \mathrm{~s}$ 3100 ft.alt. 484 E. Erythraxylum ulei O. E. Sehuly

(23) foto thrub with spreading brancher goeds from bose 2-3 m. high anching sil over or sterp hillsido, secondant cuzce thichets. Lus. 1itht yellowish green UST RO to green above, choll. pcle beneath GIt NCUT with 2 linea sometimes CoL greer. Fherits fleshy. soft, dahn wine red: US Ny at moturity, persictent. Fleshy Es GB part weass láciay leaving whitil stome, $K \frac{L x}{P}$

IAY BHE $\beta_{\text {KGE }}$ (Elleanthus is 4947 , not 4847 ) 4847. Enthraylum rimundii 0.E-fchuly

seeds to: 7 7ree $8 \mathrm{~m}$. Tall ou steep slope Wainea

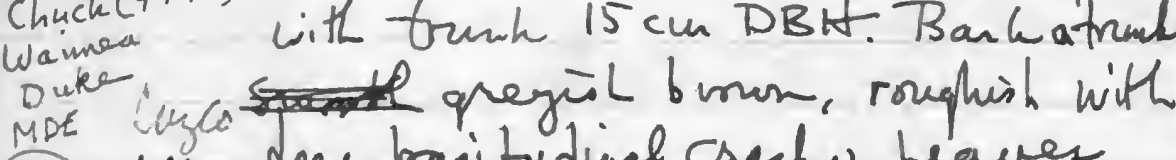
Live USM dep trajitudinal aratp. Leaves

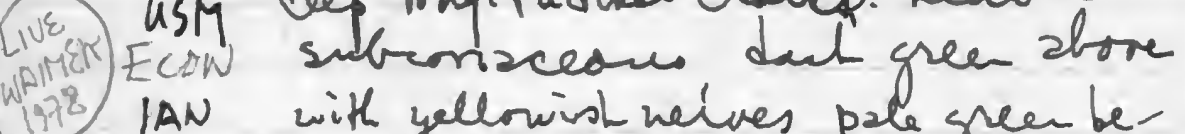
1978 IAN with yellowist neives, pale green behallyoty. E heath, nolivea, Pedices It-green, colyx yellnwish gree. Fruits deepreds resdily dofteiseing. Lus. turn prown sosn a ften picking.

(=13775 is cult) 
Clavija peruviana Stahl spinov. Isotype $2980 \mathrm{ft}$. $\triangle E T$. B. STAHL 1989

GH 4848-Clavija pungens (Willd) Radk.

limf(A) Unbranched shrub $0.5-1 \mathrm{~m}$. Retals

A) $\mathrm{K}$ yellow rouge, cente crean. Frs. bight orguge, 4849. Engthroxy hu ulei O.E.Schul.

Git (3) Shrub 15 t 2 cm. Lus. dach green. lus $\ln 2^{\circ}$ forest. Sterice. In shade.

selby 4850. Monopyle reflexa (Rusby) Wiehler ined.

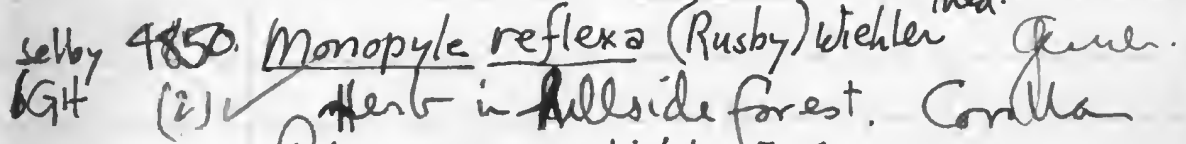
colite. det Wichlen 75

GH 4851 Peperomia

Cuyer(2) crouding ep phatic herb, spikelet pale green $\rightarrow$ madin green, young stems resty coloored.

$$
25 \mathrm{~J} \text { an } 1975 \text {. }
$$
Sahuayacu. $3850 \mathrm{ft}$. Ri: Chalpimayo.
Pacchar.

4852. Brunfelsia mire Monachino live (A) (18)

Euce Unbranched shrub to $0.5^{-1} \mathrm{~m}$. tall. USM in disturbed forest-coffee plautation.

IAN 4 Lf. rubbery, subcoriaceous, dach gren MO KOL4 2 barre, duli; pale greei beneath. Infl.

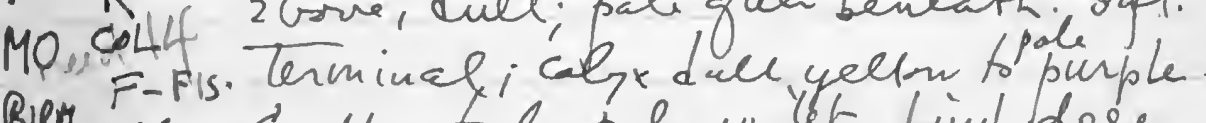

BIRH us corlla tube pale violet. Linbldeep 
GH 4857. Solsum

lugcd(2) derb. vine. Conka white. Biror Frs. pale green.

4858 . Solsumen

Git (2) kent $0.5 \mathrm{~m}$. Fs. White. Fr. ligit grae.

Git 4859. Browallia

cuyco (Aseb is shade is cafetal. Corrlla Birer (3) blue violet.

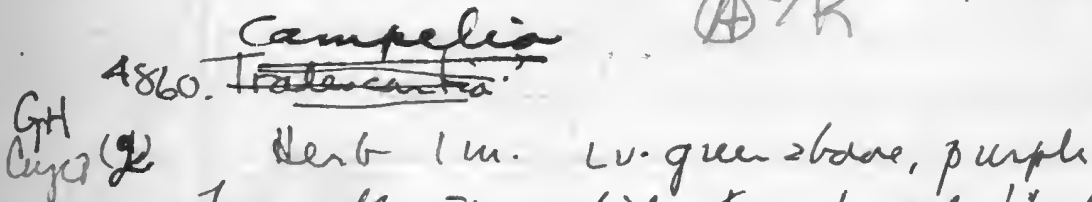
beneath. Fls. white. Fks. purple ploch.

Git 4861 fáltomata

Mo (4) Kers $1 \mathrm{~m}$ in nafetal. Crrolla Mo

(A)

GH 4862 Sanchegia peruviana (Nees) Rusby Cyef(4) Went-2.5-3.5 m. Bracts ted-orange.

Y i Corilla yellow. det. D. Uassnamen 1975 
Cup 4863, Cassia macrophylla Kunth

Cuzeo (d) Shub 2 m. in foret, Fiowers Gf pale yellowrorange det. R. Barneby 1975

(it) 4864. Denurdin

GSi (3) Erecthert-Els. pale blu-violet.

4865

Grand

AMirs (2) Terrestr. Lest. Sheath ight UST purplis. Scape purple. Ooang red purple. Rerianth crean with tiny purple dots within. Colum

GH 4866 , Pilea

Uativ.

U (2) Nent with flerhy shem swet mossy kant along trail.

4867. Regiphila umbraculiformis Mo dem

GH (1) Shrmb, unbrunched 1-2 m. Fres green. det Moldeutie 1977 .

4868.

Diastemasp.

Gesner.

$\mathrm{C}_{\mathrm{H}}$ (1) deb alang trail in moist forest.

Conlla tube yellowish. Lisub whte. det H.V Viehler 75 
4869. Melothria hookeri Cogn.

- Cucusb.

Cuyco Herb. Vine is sun along rwentauk. Crit (3) Ovany gree. Fls. white: det C. Niezgroda 1976 .

Git 4870 . Repenomic

(1) Flesh heat on moist rocks. Lus. dach green zbone, very pale beneath. Spities pele green.

4871 . Pesiflora

$G H$ (2) Uine atong trail is foret. FRs. $K$ 6-imerous, white with Cenmin red sed PTBF. septa, red toward base.

Bouchea fluminensis (Vell.) Macbride Varb 4872 . Stach tiquta det Moldenke 1975

Ny (2) plen't d.5 cu-tall slong trail.:

Pasif 
GH 4875 . Tillaudsia platyrhach is Mez Bromaliad (i) Eqiphyte. Cover bracts pale green. Upper bract + rachin bocight puns. Corolla bho-vidit det $L B$ Smith 1976 .

$4876 V$ Gim
GH(2) terrestrial fermalong trail.

GIt

4877. Oxalis spiralis R.tP. ex g. Dow

$P$ (2) Fleshy stemued herk on backs along trail. Shem red, lus green. Fls. pele yellow derandourteig

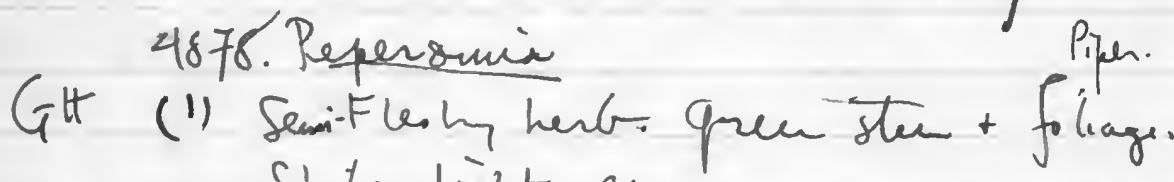
Sbrter liqht green.

4879. Lycrianther Bing Episciear

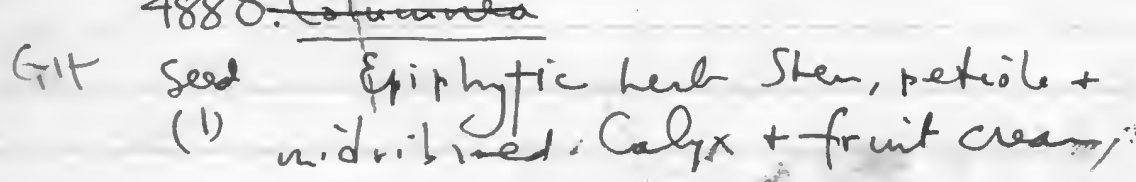

GIt $\$ \$ 81$. Micomia cretacea gleason det Wurdockil (755

US (2) thrub $3 \mathrm{~m}$. Ius grens above, white be neath Rachi + vary farmy yellow.
petses + Filanewts whete. 
4882. Miconia of multiflera Coogn. $\operatorname{det}$ wudack 1975

GH (I) - Shrub $3 \mathrm{~m}$. Stecum, petiolot loat undersides liqut rusty 6 mon (tauny). lus. green a bove. Ovary pinch. Petal white. Stzmens red-videt!

4883. Urera?

GHt seed.(3) Treelet $4 \mathrm{~m}$. Frs. bright orange. Unger Over hanging quebrada. Not urticating. 4884. Authurien eminens Schott Aroe

GH (2) Terrestrice hent on steep Lillide Mo forest. Spadix erect, bmonis pumple. cet. M-Madism 1979 .

us 4885 Pariana sp. Gramin Fuze Rhijmatoun hert in shad foret col aloug trail. Spikes gree. Anthers yellar orange.

4886. Maranta aff. divaricata Rose.

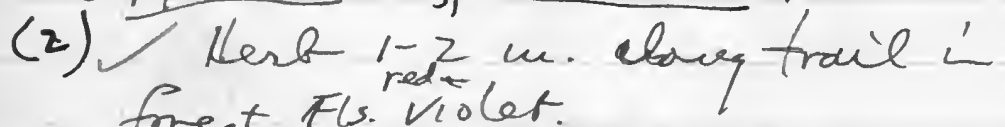

GH F det. FK 1978 . 
Git 4887 .

Cucunt.

(1) Ler vive 4-5 m. i thichet in su. Grany + calyx red-Conlla light pink. Calathea of. chrysoleuca Koern det. HK 1978

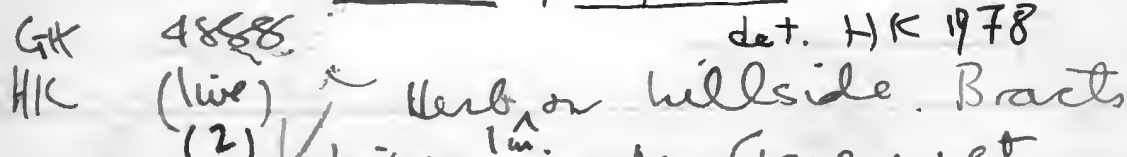
(2) Vight grien. No flowers yet. det Moldente 1975 4889. Verbena litoralis H.B.K. Elon (I) iderbidm. Fls. blue-violet. usei:dried groand lf-used for wownsh us $26 \operatorname{gan} 1975$ Alt. $3100 \mathrm{ft}$. Ue 4890. Esthosyluen coca Lam. seanders (5) Shinut cult. $2.5 \mathrm{~m}$. tall. Flsired. Chahuares. $2300 \mathrm{ft}$. 4891. Lundia corymbifera (VahI)Soudw. Bignomian Mo (2) Vine in thichet z long radside in Chahuores. Corllla 7 det. A qentry' 75 
Tperegrinum L.

van-wetertaveri 26 fan 1975. Dest. Cuyseo.

GH 4892.

4892. Troparlun webechaceri Loesn.

Cuzco

(5) Merbino in thichets alovy

$S$ sed ronts de sepals + spen It-grea. Upre lafoe

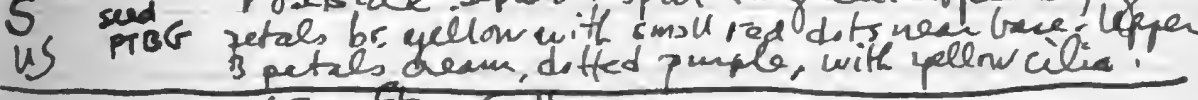
$6300 \mathrm{ft}$. Collca.

Amss 4893. Trichopilia

Ordid

$U$ (3) The on rack on steep bsuh

Foto.' 2 loug rad. Sepals t detals hight yellow green, Lif white with yellow

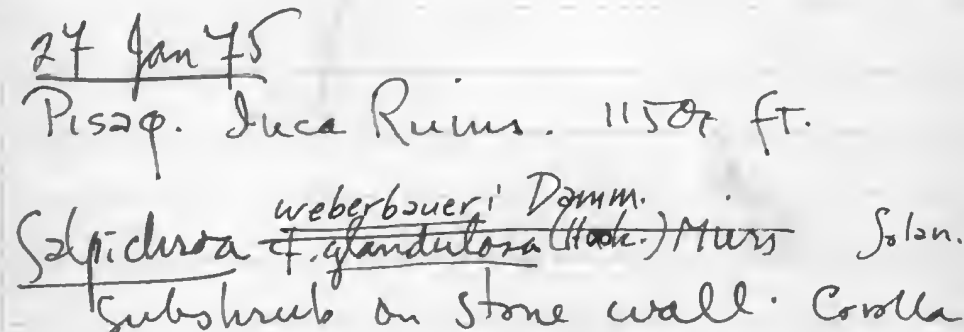

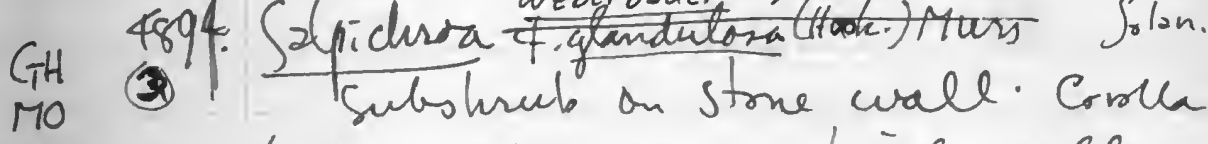
BIRM $\rightarrow K$ twbe yellow green, / inb yellow. $\| Y F(A)$ FR. green. det.s.keel 1984.

CH. 4895 . Ephedra

Ephed

USM (3) Shrub $1.5 \mathrm{~m}$. Cones yeclow.

Econ 4896. Basclla alba $L$.

Basall.

$p$ (2) Sucenlent vire. Corda yellow green lubes red tipied.

(A) $\rightarrow$ GH 
Gth 4897. Szlpichroa micrantha Benoist Sol. wall. Fis. pale yellow. det. S. Keel 1984 Pessif.

Git 4898-Bis=fora gracilens (gray) Hazms

(2) Vin in thichets. yellow queen at base, tube, sepels and petaldop pink. Fr. green.

Git 4899. Clematis seemenii O.K+3.

Renunc usn (2) Vine is thichets. Periante yellow.

Git 4900 .

Asclepial.

US7 (2) Unie in thichets. Fls. yellowgreen.

4901. Oxalio

Oxalid.

Punic. (I) Trevercled hert, Steun fleshy, ved. Podicel \& Caly $\times$ red. Crrolla yellow. 
PErcu: Depto. Cuzes. Prr. Paucartambo Beturei Huancarani and Mica. K. 46. Alt. $11600 \mathrm{fr}$. Copen radsides 3 feb 75 4902 . Vijla

Vulic. USM (3) Weed in potatofield. Bamen + GH wriggs white with purplestripes, bamen with yellow spot near base. zheel petal violet.

$4 q$ r. Sonches sp.

Comp.

GH (1) Weed i potato field. Rays yellow. sten thich with milky juice.

GH 4po4. Oxalir petrophila R. Knuth Oxalid. Herb on rock wall along strean. calye ted to greenit. Con lla yellow: 4985.Epilobium Onagr. USm (3) isert among grasses clong strean. itpanthi \& coly $\times$ darh rd-purple. Corlla pinh.

4906. Orthresanthus dridace (2) Herb sumg graskes-Ovary dark red. Perianth puch. 
4907. Costilleja

Scrph.

(2) Prstrate sumi 2 quatic hest in marshy place sloug road. Bract. pale yellow greer, ted-orauge in upher halp. det.r.Paston 1884.ex Lond (4)) $K h \rightarrow$ caiophora pentlandii (Paxt.)G. Don Git 4908 cajophord? pachylepis Urb.+gilg. WrT (4) Vine in thickets + hedgenwis F n, unticating hairs. Petals det Kew 197.

(A) $\rightarrow K$

Near Paucartanho on rad Alo Acanacu. Alt. $950 \mathrm{ft}$.

Cit 4910. Fuchsia Onzer.

USM (2) Shrebla am. Infl-pendant. Ovary light green. Hypanthin + sepale pink. Petols deep reddis pinh.

Git 4911. Cusceda

Cuscut.

us's (2) Parasitic unie on Scita Brom. steus purplioh. pls. cream. 
K 4912 . Solanum (Sect. Holophyllum)

Git (2) Shrub in oper place along quebroda, $3 \mathrm{~m}$. Corotha yellowith. seadPTBG Fis. pupple. $\operatorname{det}$ A. Chitd 75

GH 4913. Solamm luteo-album Pers. det. minee1982

USM (4) Theelet $3 \mathrm{~m}$. in thicket ollong $F$ rad. Corrlla violet WY 4914 Aegiphila mortonii Moldentulverbenas usM (4) The let 4-5 m. Fls. Creamy GII white. Frs. green. 4915. 4915. Geru
(8) Terrest. fern with hrvizontal
rhizme Lvs. $2.5 \mathrm{~m}$. Inng petiol 1 m., with 17 prs. of pimal.

USM

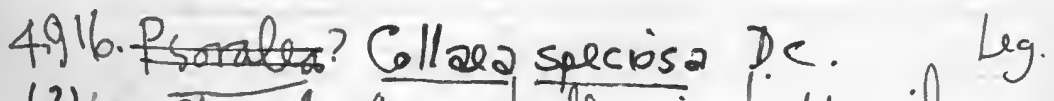
(2) Stureb $4 \mathrm{~m}$-tall in hillide

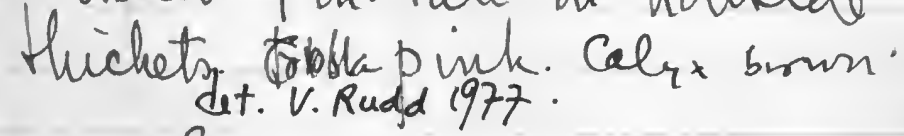

GII 491f. Passi-lora Crrlla tube It greer. Sepals dach red abavisly, punc adexially. Petals pink. 
4918.

Asclepiad.

(1) Urio in thichet, Contle pale quewing yellew with broun spit at base on $26 a x i s l$ side.

USM (2) $\frac{\text { Serjania }}{\text { Uine in thi thet. Fis-creamy }}$ yellow.

F920. Azolla

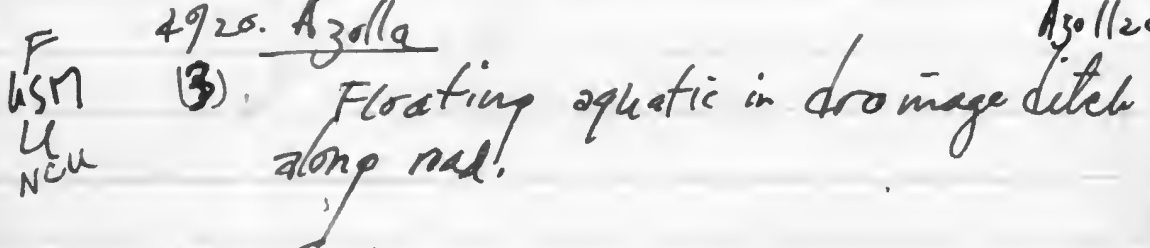

Git 4921. Slodea

USM (3) Rosted zquatic is draniags ditch u along road.

Above Pacear tamboton rool to Dbra Aeanace. 9800 f. openthecheti.

Git 4922. Bomare

Ameryll.

U. (2) Vive in thichet. Pedunde procts and pedicele quevish with dark Red. Orang red-puple: Sepals red range abaxisly, yellow

A $\rightarrow G B$ orange adaxially. Petal, yellow wange with dack red spots on adaxioly sutface. 
Come:

GH 4923. Mutisia lanata R. +P. det. E.H.Zardini 77 aSM (4), Vine in thickets. Rays bright red, $F$ yellowish at bace. Stylec red. Disc yellowish.

proboscidea Benoist

GH

4924. Salpichroa

Solan.

USM (7) Kerb-vino in thichat. Twigs. mo setioles t ajor leaf nerver red. Calyx Mo $\rightarrow K$ K tube green, lo berdora red. Corslla tube K us at thickenel simus det. S. Keel 1983 . dull ted, lokes yellowish, bright yellow

(A) - NY

1925 , Lathyrus

$\operatorname{Leg}$.

KSM (2) Vive in thichet. Calyx black. Bamer GH dephlue-violet. Wiugin white. Keel creamy white with pusplitip.

4926. Salceolaria

feropl.

GHt (1) Scandenthers in thicket. Calyx

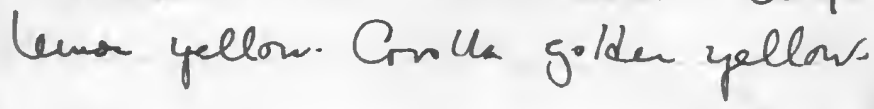

4027. Galceolario

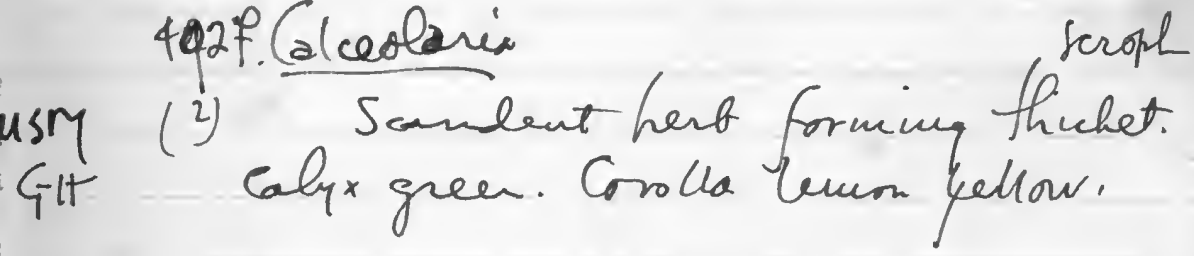


GH 9934 . Helosis Coyennensis (Sw.) Spr. Bulanophore $F$. (3) Rost parosite in thich humus. Cusces Peduncle t iffl. red.

$C$ Let $B$. Hansen 1976 .

GH 4935.
usy (2) Tentestrial fen in shade hillside usy (2) Terrestrial fern in shady hillside foreat.

4936 Asplenin?

GH (t)-Ters.Fern on steep wett hillside foret.

4937 . Parateohleria sp.

Selley (2) dent (m. generion. GH (2) Whet in shade frest. Gakx palegree. Corolla red, dull yellow within with tark red spots on lint.

GH 4938 Becleriapollidiflora Fritsch geoneriead. SEC? (3) Hent to $2 \mathrm{~m}$. Ges* 1t. green.

Corrila ovange. det Wiehler 1975

GHt 4939. Elephoglossum

USM (3). tern on steep roudbaik.

4940. Pityrogramme

USM (2) Fern on clefy ì wet forest aloug quelirada. Waxy white underveath. 
F $49+1 \cdot$ Asplemin

Lism (3) Feru on rodbauk.

4942. Anthurium

Arre.

(1) Ters, herb in wet forest.

cus. dark gree, rugose: Spathe 1t. gree. Spadix green.

4943. Eidendme Orchid.

U. (2) Qrehid on step que rosdbant.

Ames Calyx +corlle deep pinh. Colm red duch. Lip lokes pinh, white at sente with yellow spot.

4944. Efidendru

Orchid

Ames

(1) Orchid n steep ope radboul, Gelx + Coroll deep schuon pinh. coln + lip same. Lip with drangs callen.

4145. Epidendru

4145. Epidendru
Ormes (2) Orchid.
Al. Bure whit. Ames Flo pure whit.

4946.

(1) Qrchid m steep oper Lillider. 
Fis. pale puih, lip with yellow spot.

Ames 4947 . Dleacthus

U (4) Orchid on steep ope hillid. cuses Bracts traqenta pinh Fls. prich " near base an infl." White abou.

GH (1) Segonie

Ordid. Orasy pile green turnum red. Petraith abaxisly beep pinh, 2daxially pale pich.

GIt 4949. Crotolaria nitens H.B.K. Surph.

Uuco (3) Nest $0.5 \mathrm{~m}$. on roadside. Calyx pale lonom. Corrlla pale yellow, bayner red adaxis'lly.

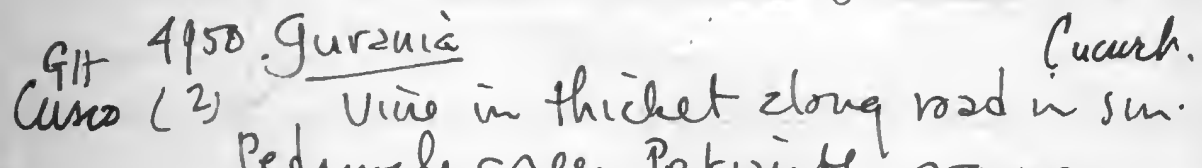
Pedumcle green. Petianth orauge.

GHt 4951. Eupztoricio

Comp.

us

(3) Went l.o m. ounitrostbanh. Fls. pala blue-violet. 
det D. Simpson 1977.

Alchornea pearcei vel. aff.

Euphnol

Tree $8 \mathrm{ch}$. on hillside.

Frs. green, with red 6 kus.

stiguas red. det D. Simpson 1976 .

4953. Desmodiun molliculum (HBK.) DC.

USM

(3) wet rodbant. Vireng hert calyx red. Cordua purphas blue. det. T. ploweruen 1982.

4954. Erechtites valerianifolia (wo f) $x$ / Lactucene

unicate (1) Nert on hads de. Fls. Leep pinh. Det E. Zardini 1977 4955. Anthuruin

Arac.

GH Ters. hent an ope pad banh. LV. Coriceoum. Spathe It. gree. Spadix redisl.

Git 4956. Munnoziagegantea (Rusby) Rusby
us (2) Nerb $0.5 \mathrm{~m}$. on wet rozd:

(2) Nert $0.5 \mathrm{~m}$, on wet rod banks. Lus. correred with white indument. Fis yellows. det. H.

(R.t.P.)A.C. Robinsonili) 79

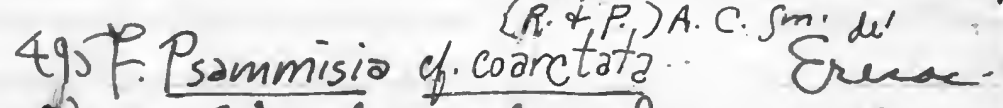

Cuy

(3) Standet shruh ou wet

GIt open rodbank Pedicel, ovary, 
upper $\frac{1}{3}$ + boes white.

4958. Physalis

Solen2e

Gi(2) liest $\mathrm{m}$. on radside. Caly

BHRA $>K$ green Conlla 1 . yellw with 5 dh. brom spits at base calyx in frint yellow-gree $F_{R}$ - dull orang.

4959. Ly spodium

Lycoped. "lezes (2) Vimin heit prostrate on oper
GAllside.

4960, Vismina

NY (3) The $8 \mathrm{~m}$. Lus. nubbery, rustyber-

Cuyeo heath. GOx It-gree. Corllla crean. Filzenents hulite. Ovary orang, stgles

4961. Sindax

U. (4) Wert. Uime $8 \mathrm{~m}$. tall-Ripe fricth dork trauge-sed PTBCT

Git 4962 . Poperonia

GH (2) S Succulent épiphytü herb in wet F pret. Spine yellow-green.

4962-A.

Discompate Thallur yellow. 
Cuseo 4963. Piper GII (3) Sriter $5 \mathrm{~m}$. in wet forest.

4

GH 4964 Pipen

Piperac.

surub $3 \mathrm{~m}$. Lis. rubbery. sithe pale green. Fin. Wimi

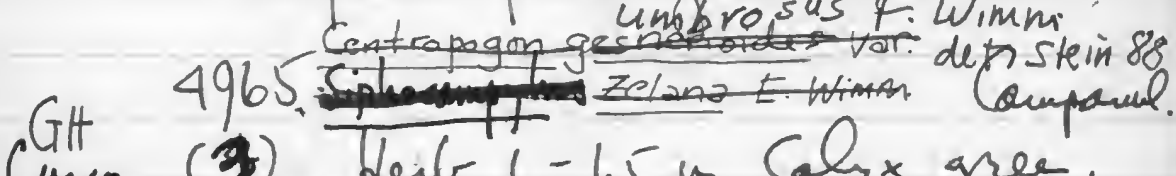
Coyo (3) deib $1-1.5$ u. Calyx gree. forest. det C. Niezgrola 1996.

G* $4966 . \frac{\text { Piper }}{\text { She }}$

Ifiles pendant, dart green.

Piperac.

496 f. Dequia parviflora $P, \&$ \&:

Gites (5) Wenf to. $4 \mathrm{~m}$. tall is wet us forest. Perianth white stames us ycllow. Ovan gree white.

K sedPTBR Petiole with Ned excrescences. us. Fuddich beveath.

4968. Psy chotria sp. aff.P. albertsmithii Rubion.

(2) Slurub 2-3 in. i wet foret. Rechis fruits graen. 
GIt (1) $\frac{\text { Croton }}{\text { Tree }} 20 \mathrm{~m}$. tall, trunk $30 \mathrm{~cm}$. Reraith pole gree Ovary reddich.

Git 49f0. Anhurim amoenum Kunth. ex Schott us (2) terr herb wo wo fort. Espathe det Den Nicolson 76 .

497\%. Nephelea cuspidata (Kze.) Tryon (9) Treeferin with trumh $5 \mathrm{~m}$. tall. Leaves $2-3$ mi Pinnal 30. us

Cusco 4972 Pilea

urticae.

Shruh $1.5 \mathrm{~m}$. Infl. gremid. 4 yellow.

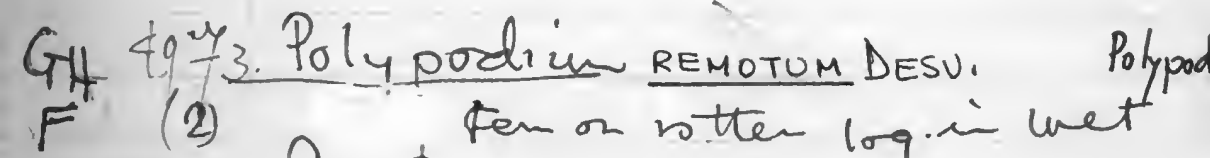

(2) forest. Fen on ittter log. in we

4974. Miconia sp. Sect. Cremanium Acolestom

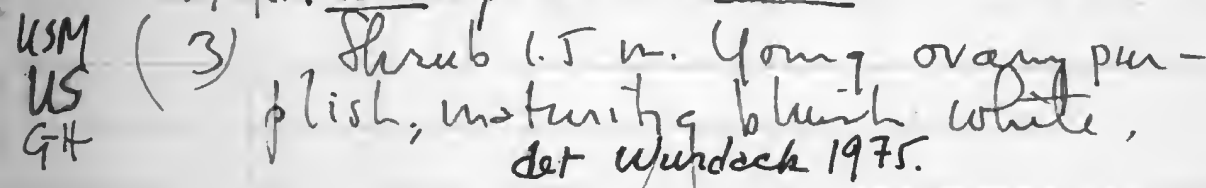
det wupdack 1975.

Git 4975. Siparuma (2) Shrubs of m. Fls. yellowish. Frs. red. 


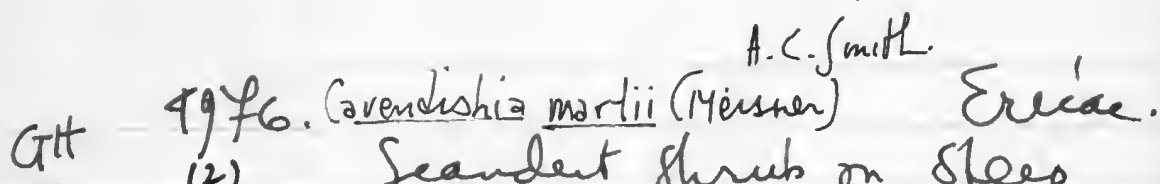

F (2) Seandent shrut on Steep Oen radbank. Rochis reddish. brary qrees. Corlla cream white. det. Lutegn 1982 GH 4997 . Rernowa of jussieuana Mart. Cosco is wet frost elm quebrada. BH Rache grez, red at moturity.

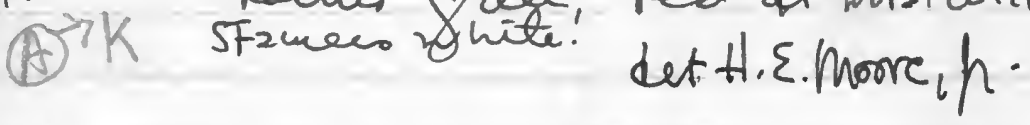

Cusco 4998. Piper augustum Rudge (?) Aperac

u

Gr

(4) Tree let $3 \mathrm{~m}$. Spilie gree. Lu wet forest. det.w. Bunger 1981.

GIt 4979 . Nautilocalyx sp.nov. Genciad,

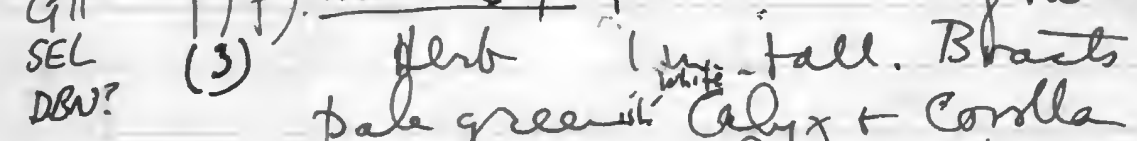

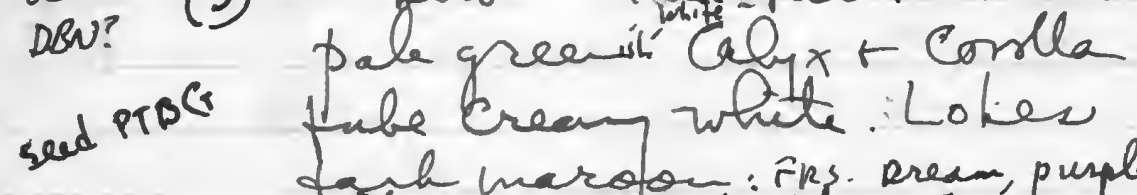
tube creary white. Lokes

4986. Peperomia

GH (2) Elestyherb ters. Lvi.

Piperac. redish inderveate. Spibe ple grean. 

Kosxi_pata Valley
4 Feb/75

4481. Neliconia subulata R. + P.

Muser.

U (2) Ast $2-3 \mathrm{~m}$. Fall in wet forest.

Glt pachit broct red, narmoed puper part gra-Pedicil red.

Oveny gree det. Lerianth andersson 198 ellow.

Cemponal

GH 4982 .

Nesb I un. An open roadbouks. Calu, dach purplist gree. Corlla rel-orauge. Gi 4983. Pitcairnea oduntopoda Bakes B muclice radtiank. Rachit Cabyx 1t. grae Corlla ied. $\operatorname{det}$ L.B. Smith 1976 ,

A17. ft. 5 feb 75

ctt 494 . Reurhini thyrsoidea (R.+P.) P. +E. $U$ (2) kert $3 \mathrm{~m}$. tall on steep.

diff along quebrada. Bracto red.

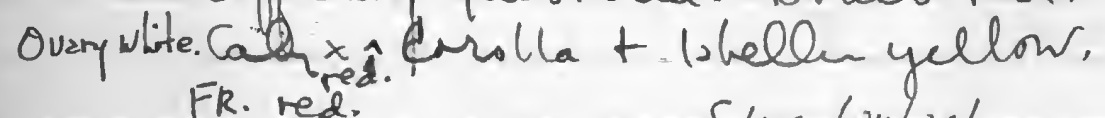
FR. red. Solms-Laubach 4985. Hedyosmun racemosum (R.+P.) Chloridh GH (2) $\begin{aligned} & \text { Thee } 6 \mathrm{~m} \text {. Fls. grea. FRs. } \\ & \text { whte. }\end{aligned}$

det. C. Todzia 1986. 
4986. Parakohleria sp. Geanen.

GHt (1) hent In on steepcliffic wet for est. Corllla yeulow.

4987 . Certropogm yungasensis. Camponal.

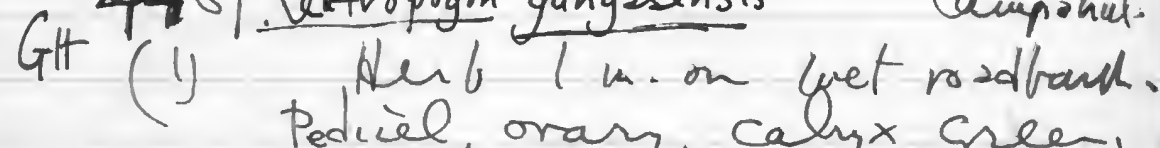
pedicel, oray. calyx green. Corrlla red orange at bare, gradug yellow orange to wards tip. $5000 \mathrm{ft}$. det.

4988. CYATHEA
usm (6) Tree fern with trunh
GH 3 m. Teaf $2 \mathrm{~m}$. with 34
$u$ pimmae. (Rusby) wiehlen DBN 4989 . Corytoplectus riceanus Germen. Stl : seed ther I m. Lis. daif sreer GH seed bave redpurpla below.

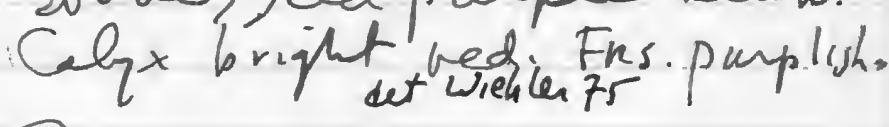
4990, Pityrogrzuma EBENEA VAR. AURATA

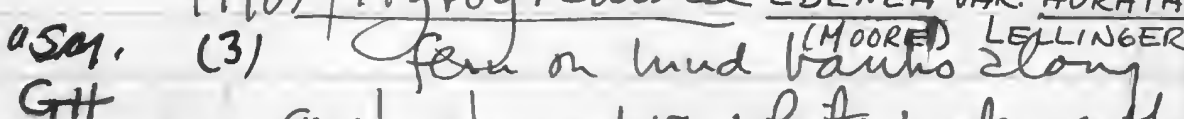
US quebraba. LUS. white underueath. 


$$
5 \mathrm{feb} 75
$$

4991 . Stromanthe sp?

Marent.

(A) pal Hest to 2 m. tall along $u$ (t) quebrada in forest. Bracts USM

开

GH white. Calys whet. Corlla white. Lakellum purplis. Ovary orange. dat HK, 1978 .

4992.

Thigmeleac.

GH (1) Treetet $3 \mathrm{~m}$. Fis. yellowish. Frs. orange. Los. dank green
zbove, whitish beneatl.

Zingib.

G4 4993. Renealmia thyisoidea $(R+P)$.$P . +E$.

$u$ (4) kest 2 untell. Practs red. Ovany white. Caly+ red. Stere redchish boun.

Cuscos 4994.

Aralian

GH (2) Troel 2-3 m. FRs. Creanwhite.

ATt. 3\$00 ft.

4995. Meriania hexamera Sprague det wundech1971

us (4) Shrub $3 \mathrm{~m}$. ven deff merhauning Corlla magenta - puinh. Stamers gelbur 
Alt. $400 \mathrm{fr}$.

(3) Nenb 2 m. on steep wet rad-

Cusco bank. Bracts qreen wate brounsh GIt margin. Brattelesigreenish Olyx crean. Ovary creany yellow. Caly pinh, lokes pale green. corolla yellow-orange. Labellu vel orange, lip deep yell mwith teep jellow stripe. Stamen pale yellow.

PElru: Dept. Cuzco. Prov. Pencartbubiol. Pilcopata. 6 Feb 1945.

Ailt. $720 \mathrm{~m}$.

Rósd Pilcopata-Patria (twuado Peneartanba), nean Pilcopata. Sliatlly distrubled 1 " forest.

US 499 F. Tococa quadrialato (Woud) Machile

GIt (2) Shmb I m. Calyx pale yollow grea with pinth hairs. Petal pale pinh. Ovany in fricit dork purfele, calyx red. $\operatorname{det}$ Wudack 1975

Monachimo 4998. Schindleria densiflora (0. Ktye.) Phytobecac GHA(3) Herb is forest, $1-1.5 \mathrm{~m}$. Lus. Cusco gyeer above, with veluet sheen, br. red-puple

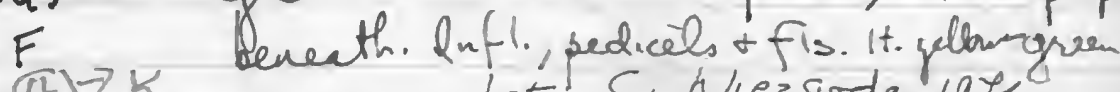
(A) $\rightarrow K$ det! C. Niesgoda 1976 . 
4999. Palicourea subspicata Huber Rubiacan

GH (2) Mubvanched shrub $1-1.5^{-3} \mathrm{~m}$. in

$F$ forest. Pedumcle dack binumise grear. Rachi and pedicels ret. Hra cy red. Gax ved! Fr. dauk gracu.

corrle yellow. det D. Simpor 1976

5000. Brufelnia mine Monzchino. Solenze.

GHt (A) (3) Subshrub I m. tall in wet shaby. Frest. Lus. dach dell green abre pale yellniul bye foding to pale Violet. Calyt crean.

5001. Leandra secunda (Don) Cogn. Melastom.

(3) terb in forest understory.

USH Car It. gree. Fis. White. FR. dark purple US blt blach.

5002. Lyeisithenglandulosa (R.tP.) Bitter Solonce GH (f) Vin in thichet in low swamey forest Frs. bright red-orcuege.

GH 5003. Dichorisandro

Conumelinac.

U (3) Heb. vine in $2^{\circ}$ foret. Conlla F wlite. Frs. green

cuet: AA

aff. $\rightarrow$ Psendovgitehiana Kennedy

503-A. Golatheariveitchrans Hook. Lus. dian-

Five The 1-1.5 m.tall. Sterile. Lus. diarphotoss hive taceres, punple beveath, pottern $1 t+$ das green 
Myrosma cannifolia L.f.

Git 5004. Myrosma dettik i978. Mrrant HK (2) Las herb in foreat. Bracter ligit green. Fls.creamy white?

5005. Piper augustum Rudge vel sp. aff.

GH (2) Iterb I m. alore $\frac{1}{d}$ il in forest. Peduncle red. Sfile cream.

GH 5006. Solamum

Solmer

(1) Corbla greany yellow. Lerb/ $\mathrm{m}$. tale is forest.

5007. Piper tectoniffolium Kunth. Piperr

$F$ (3) Shrub $2.5 \mathrm{~m} \cdot 2$ (ong trail in

Cusco foret. Spike crean, greevis i GH frut. det. R. Callejas 1982.

GII 5088 . Vive in ofer thichet along rad.
$K$ (2) Vive K (2) Vive in ofe thichet almp rad. Steivith mity latex.

5009. Bauhinia glabra Jecq. Sensu Wunderhin CIH (3) Tree $6{ }^{\circ} \mathrm{m}$. tall on rodside. Cusco Caly ligut green. Petah white.

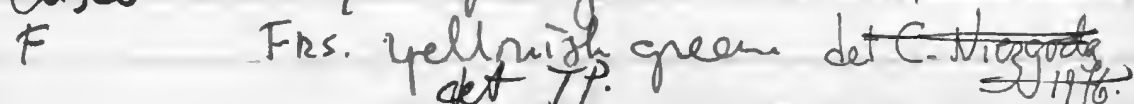


Gram.-

5010. Cryptachloa unispiculata Jaderstrom Banbusaid.

Us. Kenc in foret suderstory.

5011 . Costus acreanus (Loes) Maas

Zingib.

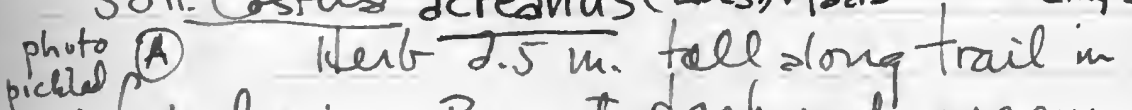
seed fst. clearing. Bract, dash red, greew hear tip on s baxisl sunfoes. 'Brackesles

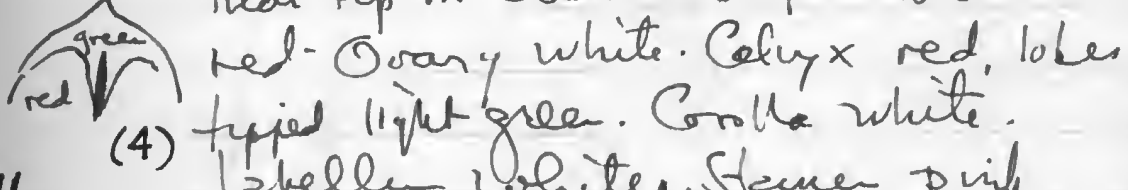

USM

if. ventricosa

5012 . Iriartea sp.

Piluae

(B) Tree $20 \mathrm{ch}$. tall in forest. curco. Sheath $2 \mathrm{~m}$. Lus. $5 \mathrm{~m}$. long. Infl. 2xis

BH palt blackea. Frs. light grear, maturity

5013. Renealmi breviscapa $R_{i}+p_{i}$ Zingib.

(1) (5) Kent $2 \mathrm{~m}$. tall in forest along tracl.

$u$ lufl. axio liqut yellow list-green knath

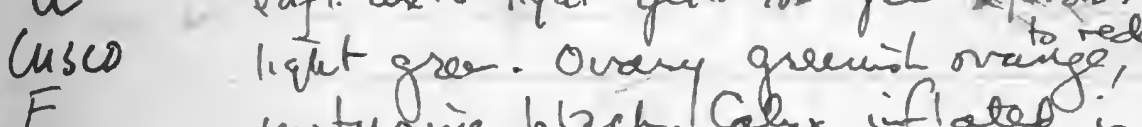

Fit unturing blach. Coly iflated in

GIt - fruit; orange Arit yellow-trange.

US Seado dark brom. 
Centropogon Cornutus (L.) Druce $50 / 4$ det C. Niezgoda F6 (2upanut.

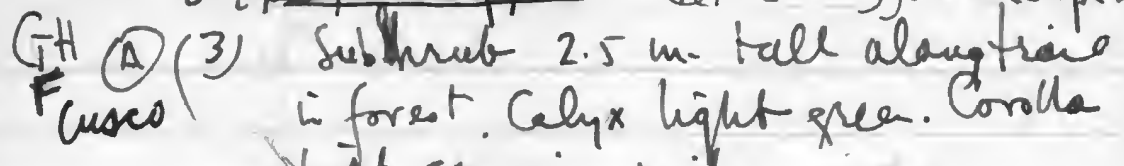
vigut carmine prit.

5015 . Pariana

Gram. -

Bombusoid

US

(I)

kerb in forest floor. Culus s-mint purple.

gever IMT)

5016 . Colothea aff. peruviana

Throut.

foto Herb 1-1.5 m. tall. Bracts pals

FI(A) green with cream margin. Bractedes

GH hyaline pink. Calyx white. Corlla white abaxialy, white with purple stripes sdaxiall; stammide pale yellow. Lus. hed-green abate, paler grey green beneath.

5017. Monotagma laxum (P. + E.) Schumann

(3) Herb 2 MET. M. HAGBERg 1990 Marent.

(3) ker $2 \mathrm{~m}$, tall in forest. Lus. papyfoto tacesen, shing dank green above, lighter

(A) $\rightarrow F$ green shing beneath. Pecuncles gren.

u practs yellow green. Calyx hyzhie pinh Coritatube white, tobes pala grea. Stami. nodes + St zuman pale salruon purk. 
Nean Pilcopata on rad to Villa Garmen. $\partial^{\circ} \mathrm{f} 5$ t. GH 5018. Pipen longestylosum C.DC. dect d.R.Callejes $F$ (2) Substrablim. Spiterect 1982 2. Subsarab th-Spines greemsh. 5019. erythroloma (Lwbg.) Wieklew SEL (2) Vine in thechet zloug rad. Bracts Glt? dark ref. Cols daib carmin red. Corkla waky yellow, with red tint on louner 2 labes on inside. Fr. dorl red.

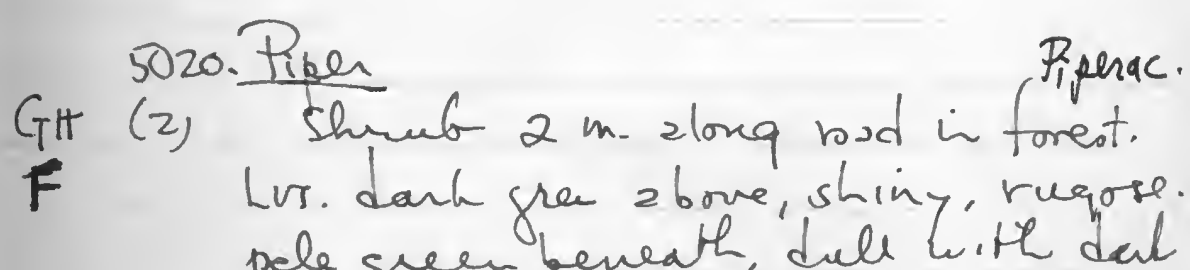
pele greenbeneath, dule hith dat buon-purple nerves. Spcter erect, pale grean, It broum at maturity.

21. Centropogon roseus Rusby det. B.Stein 88 GH Sor anulac $F$ foto. Subshub $2 \mathrm{~m}$. in thichet dopp. (2) road. Caby It. grea Corrula 'pink. $\operatorname{det}$ B. Stein 1985.

5022. Clitoria Ap. (not matchat at $F$ ) Legumimos. GIt (2) Vine in thichet zlong roed. Pratts yellow-green. Calyx rusty brom at bove, crean towards tip. Baneer brossish fourth. Wivep + heil creany gellow. 
1. 5023. Miconia nervosa (Sir.) Triana Melastam, GH (I) Shrub $2 \mathrm{~h}$ is open thichet 2 long: rodd. Rachis red. Orany yellow green turning red. Fls. white.

5024. Pipes

Piperac.

CY't

(2) liqut brown.

Treelat $5 \mathrm{~m}$. Spetes evect: $\bar{F}$

5025. Parian?

Gram.Bumpoiv iv

US (1) Went in thichet aloug traile. Calues reddric at base. Spate hagit green, (same as 5038)

5026 . Wathea 5 .

Marsutac.

(2) A if Nent ifu. tals. Bracts dule " GIt Sracteoles hagline, crean. Colx creamy white, lobes tiped mirple. Grolla yellow. Strminde t Hamen yellor.

502\%. Costues off. quanarensis Rusby

u

(i) Herb $3 \mathrm{~m}$. Tall. Bracte ted below, yellowish greensbove. Oron White Calyx red. Corrlla jale red-riangs: (prinst). Labella + Stamen red-orauge. 
Monotagma PLURISPICATUM (KOERN.) K. SCHUM. 5028 DET.M.HAGBERG 1990 Marzent.

(2) Nerb $2 \mathrm{~m}$. tall in forest margi (A) abry trail. Lus. darl green zbove,

GH $F$ ? poler grey green becuecth, dull'bith sides. Pract pale yellow-green.Flsi calyx hyoline. Eoplla pale purplist btown. Labellew creany whate. Stanow purple-6ms.

5029. Costur off: guanaiensis Rusby Zingib. $u$ (2) Hent $2 \mathrm{ln}$. Ligul ted-6rown. GII Bracti dull red-brom bevect, expred part light green, wrinhad. Ovary white, Glyx red. Corrlla yale pinh. Sabellu pele pinch with yellow stripe

Stromanthe confusa Schum.

5030 Marent.

U (3) Werb $1.5 \mathrm{~m}$. tall in foret margin.

Ift (A) Bract green with hyalin margin Colx pla green. Cor Ul white. Staminale white with blue viilet way gì. (As. small) white with 6
Peullinia

5031. Serjouria Sapind. GIt (1) Vine in thichet. FR. car nume rd. Seed blach. Aril white-

5032. An thurium

Arac.

Git (1) Hub on ground in forest mergin. Lnfl. erest. Spathe It green. Spadix b muswirh 
5033. Mi conia aurea (Don) Naud. Whelastom.

USM (3) Thee $6 \mathrm{~m}$. tall in foret margin Cusco 2long rood. Oran white. Calyx pate US pinll, tipped green. Conolla white. Glt Stameen magents pich.

Marent.

F 5034 . Hylzeanthe unilateralis (P.+E.) Jonk.t gonk. $u$ (3) Herb I m- tall in forest may i HKt (A) Bracts it-gellow grean Calt Git hyalin white. Conlla hidinite, lo les CoL immispurple. Staninode white below, lim'b yellar. det HK

5035. Calathes of.peruviana Koern. Marent. (3) the $0.5 \mathrm{~m}$. tell on forest

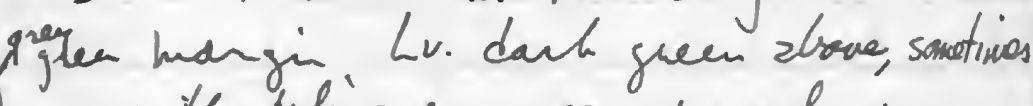
with sale' grey-green un wirs

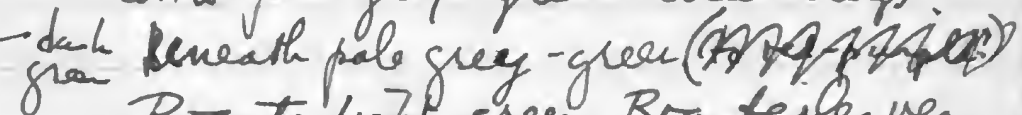
gra Bracts l, 2t grear. Bracteiler vey pale green. Coly pale yallnw-great. Gon la pale cheam. Staminode giden u. yellow det HK 1978 $H K \leftarrow(A)$ GH $F$ 
f Feb 75

Pilcopata.

5036. Bremfelsia mine Monachino Jolnze.

(3) Unbranched Shrub I m-tall.

GH Lur. rubbery, coreaceous, darh green $K$ (ex cult.) 2bove, dull; Gellow green beneath.

$F$ (ex cult.) Bract lanceslat, yellow-green, eurt. F Pedicèl t calyx cream with subtle. cuttings to puplist hui. Caly x lokes convex, 2 yon Ant 1982 . Fobes extendry partially or all the

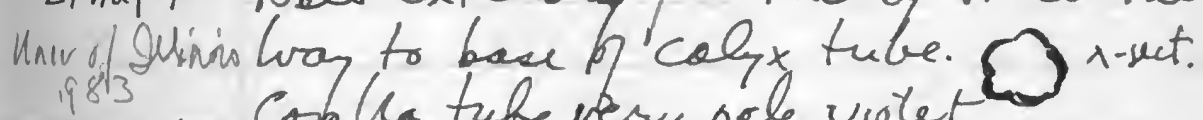

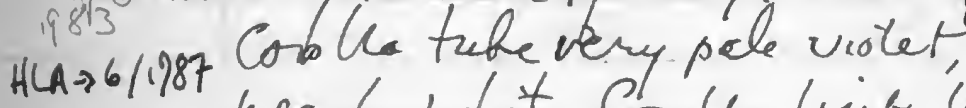
hearly whit. Conll Lint 'very pale viotat Leneath, pale violet ¿tove with white spot at centen, 8 mun.dizmete. (= Plowman 12023 in calt.)

Villi Carmen

Dol pescapracifolia Britt, F 5037 Bytheria Steral. cusco (z) Vine in thichet. Perianth pale GH green. Stamem yellaw. det.Tplompen 1978.

us (1) Ho38. Pariana

5039. Pentagonia 5039. Pentagonia (2) Unbranched shrub $1-2 \mathrm{~m}$, Stipule
Fed. Calpx red. Corolla yellaw. 
[D. loretense Krzuse det.r.B.Berer]

5045. Dracontion longipes Engl.

76 .

Git (4) HerL Petiole $2 \mathrm{~m}$. long, Cusco with migit shahe lite pattern. us Lus. ternate huch divided. spethe diak puple-6rom. spadix derch brom.

MuAprede Dias: Prov.Manu. Read from Pileos to Shm tayi, s. Feb f5. Halaya-Saluzcion 65om

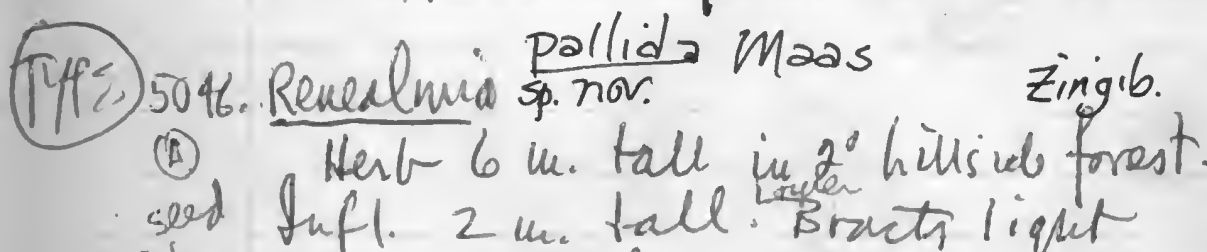
GH foto quen, spechled red. Upper brants USM (3) ple pinh beconing pale croming "Ablotypeyellow to greewich. Bracterles truards base. Radelin crean white. Ovary cream. Celyx pale crism-pinte. Corstle pale jellow. Tabellu phe gellow: Ffuits very pafe cream, celyo remmats white

live: ¿l A|

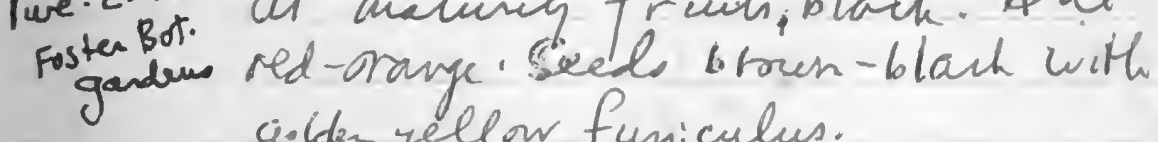
golder yellow funiculus. 
ssp. thyisoided Eingit

$504 \%$ Renealuna thissoidea (R.tP.) P. tE.

1) Herb Aturen. Tall on stexp

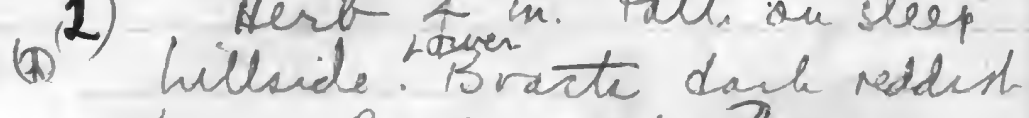

U. foto 6 mm. Rachiparih. Bupper

GH cufl. Irusts ipite-orauge. Bractesles tis. Pedicel puih oveny white. Clix an nuis pinh near hase, yellow orauge towand opex. Contha tabe pich in lowen half.

teep yellow tovand opex. La bellm \& St inew teep yelbr. Yoruy

E. Eeijoi Berg

Cit 5045 Eugenis aff. E.cuspidifolioDC.+ myrt MicH(2) Everub 1. 5 in. in 2'forest. Fre. canlifiorous on stem Fo subsp.qutierrezii (O.kuntze)rinas

5049. Dimerocestar strobilaceus O. Kunrze

(2) Wert in wet plare drov road

(A) 3-ipm tals. Brath liater yellox.

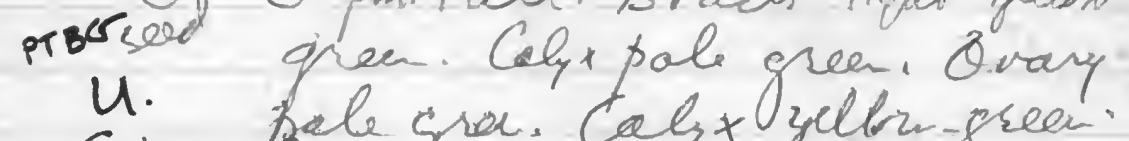

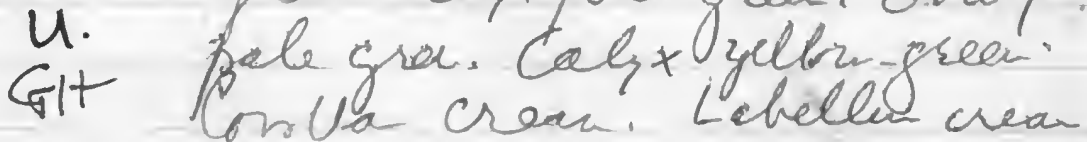


beweath (abaxially), light yellow adaxially with quidén yelno hair. stamer cream, apicel sppendage Tale yelor.

505\% Leandra dichotoma (Don) Cogn. Melestom. USM. (3) Shrub in suampry forest along rosd. Lus. dark green sbove, pale + avary with red hasin. Petals + filaments white. det Wendack 1975.

5051.qurania Cucurb.

(1) Uine in 2 forest or Tublate. Aypouthive + sepalis bright ted range. Petcle teryellow, Atuthers

Cream.
Nean Bilco pata on rad to Shintura.
2. Marent. 5052. Calathes gigos Gagn.

(5) (A)-tk derb on rasdride in forest margin UsM fit 2 m.tale. Ls. yellowish green above pale gregish gree. Pedincli lippet green. Barata Fight green, yellow orange toward base, finh' at mangin practeoles cannime pinh, yellowish trand 
base. Pedrcil yellow. Cahx punt. greenial yellow at tip. Corill tube creany yellow, bhew pale greeninh yellow. Stamiode creany yellow. with pale purple spot hea sfex on abaxid surfor

Peffu: Dept. Madre de Dios. Prov. Mzuu. 9 Pevereins 75 . Sauth A shintrago 5053 . Plipeded low frest elón vad. Piperse.

GIt (1) 9 Hert $0.5 \mathrm{~m}$. in swampy 5054 a costus sp nor. Cult. Hairand Forest, Petenham, Mass.

beneath. sterb $1 \mathrm{~m}$. tall with 4 stalks. Leaves dark glew 2607 , dark red pumple, hains whitein on both sides. Breets firm, light green whive Engib exprsed, red bzsaldy ffendage ar ar

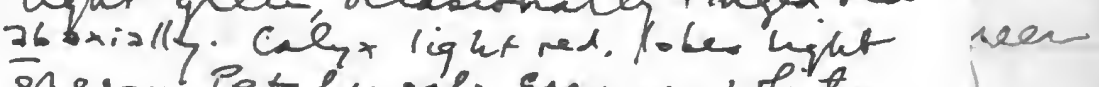
gren Petale pal frearny white. Webellew tube creany yell on lmi 6 whit, struied with red, chite central id. yellow stripe saxially. Stame apreadage 'en, teddex with adaxially yellas stripe belon

Tip Howene 6 FeG 1977. F'AA,U, Klach.

Lwe: SI Al, Hart, Selli', U.Chic, Lyow $=13044$ 
base. Pearcil yellow. Cahx punth, greenise gellow at tip. Corll tube creany yellow, to hes pale greenish yellow. Stamisde creany yellow. with pale purple spot thea spex on ab axid suifor Hamen creany yelkn.

Pefzu: Dept. Madre de Dios. Prov. Mzyu. 9 fevereins 45 . Sasth \& shiting on $r$ ad to Sulvación. Alt.c. $600 \mathrm{~m}$.

53. Piber low frost eloug road. Piperac.

(1) $0^{\text {Heat }} 0.5 \mathrm{~m}$. in smanepy Forest. Lvs blachish green above, snuewhat shing, red-purple beneath.
sterilo.

5054. Costus nor.

Zingib.

U
USM
GH

(3) Henti in. tall. dark Pruple: Lis. dach to lach geen Llowe hath ligitt brown ho ir-o. Shmy beneath, dach wime red. Bracts light yellowith geere, hed found base. Oovary white. Calyx red. Seed, \%lach.

Leve: SI Al, Hout, Sell'y, U.Chic, Lyow $=13044$ 
5055. Solanum

Solonze.

alzco (2) Shumb 1 m. tall. Less SubcorizGH Gown. Fks. crean with dah quen reticulate patterw above Cutones Forest on steep hills ide 2 long qnemadx Econ 5056. Brmfelain mire Monxchimo Sterile.

Cusco (y)

Rosette herb $1.5 \mathrm{~m}$. tall Cypers

GP brum.

G+ 5058 Parianasp.

Grim.

Bacubrason'

us

(2) gran on forest flook.

det Soderstrom 1975 Flacount.

GH

5059. Lunania Parviflora Sprua

.

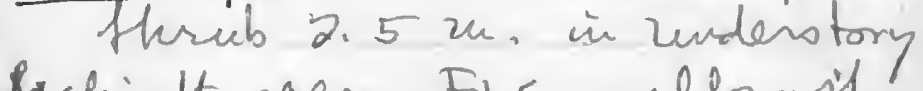

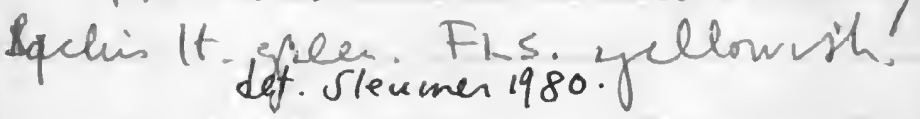

5060 . Calather aff microcephala $\operatorname{det} 4 K 1978$

F

(I) Herb in forest. Lus ved green sbove, pale green beveath. Bracts 1t. yéllow grean.

Git 5061 . Besleriass.nor.

Gemeris SEL (3). Henb / m. Rachi grea. Podicel det wiehtellow orange. Caly x orange. Conlla orauge 
5062 . Geonoura of. brongniartii Mart Palm. $^{2}$

(b) Sucll patu trumk $1 \mathrm{ft}$ tally Cugco Las. $2 \mathrm{~m}$. Rachis yallow yreen BH furminged. Frs. pole grean GH turinitglack. det H.E-hoore, q.

5063. Codium

Arac.

GH

(1)

Terb, acaulescent, pedunde spathe gieen. Youny fres.areany

Guzco (2) $\frac{504}{\text { Paullinia }}$ Vin in thichetr. Fruit rod. Git

Leed (1) Treclet 3 m. is understor

GIT FR. yellar Mesocarp whit. gelativons matrios.

dow 5066 . Calathea off. undulato Marst.

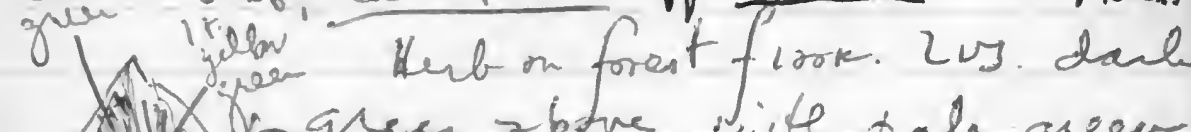
(i) Green 2 bre with pale greew

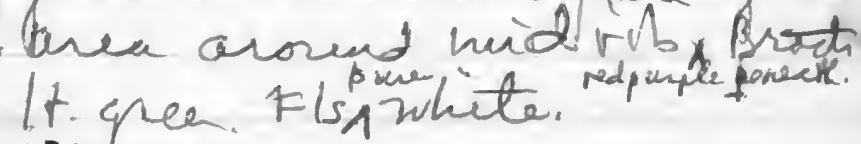

(3) $u$.

gangrean two $\mathrm{HK}_{\mathrm{GK}}<(\mathbb{B})$ 
$\log 2 n$

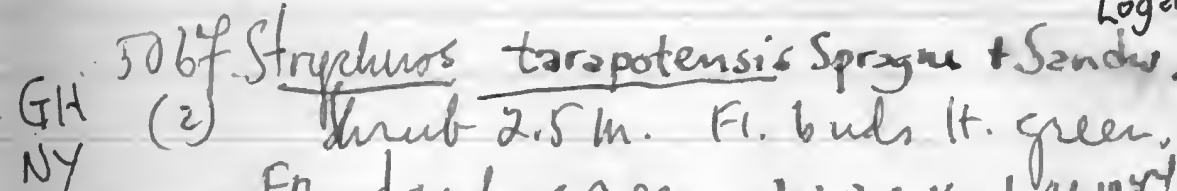
NY Fr.dach green $\operatorname{det} B$.A.Krmkofy 1977 .

GIt jo68. Guramia

Cucurb.

(i) vine in thichet Hypanthi orange.

5069 Psychotria trivalis Rusby Rubiae.

USM

(3) Trealet $6 \mathrm{~m}$. with spreadin bramches. FIs lewor yellow.

Melest.

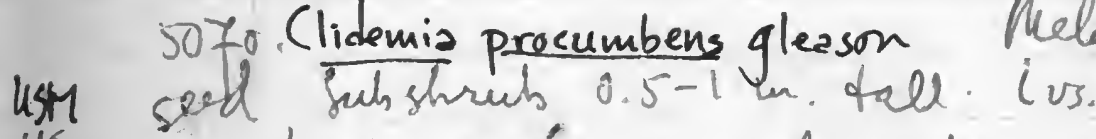

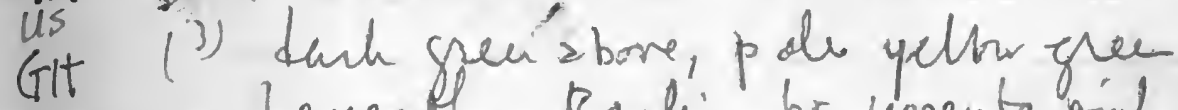
beneath. Rackin br. wagenta pinh. Frs. pole bive, det Wurdack 1975

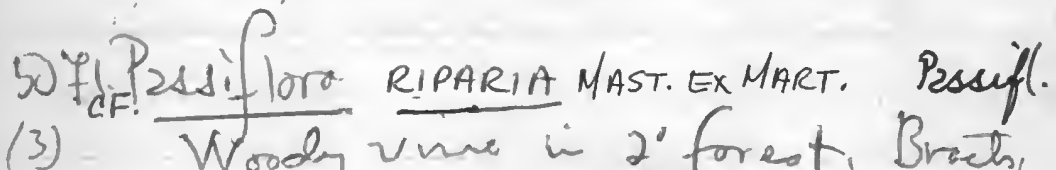

Unco (3) Wood unve in 2 forest. Bractsi Git colyx + correle areany whit, tinged F with pint. Corsua banded whiti with dart wite red. DET. J. E. LAWESSON + L. HOLM NieLSEN 1987

USM $50 \mathrm{~F}_{2}$, Paullinia alata Sapund. FFH (3) wordy vim is thichet. Fis. coulifloms, creany white. Fiess 
5873. mendoncia glabra (Poppt Enel.) Nees US (2) Unie in thinet. Brant light crean Coplla crea white. det D. Wasshemen 1975

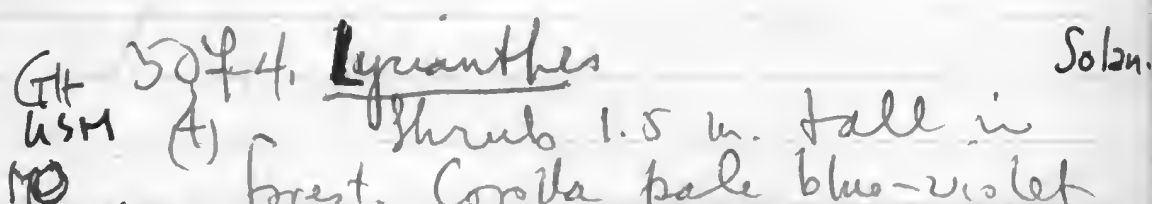
BirM $\rightarrow$ K frest. Corolla pale bluo-violet.

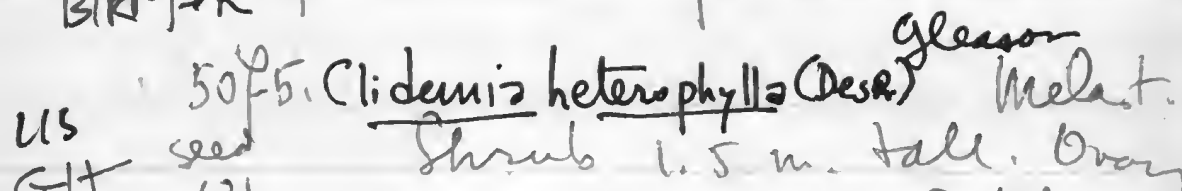

(3) tpedicel magenta. Petalit filaments wijite. Fis. dark blue. (Axillang $f(3)$ det Wundack 197\% 50 f6. Rleodosipathamukuntaria Croat Arcc. GIt (1) Ceriting epiplatic lent. spathe creany white, (PARATYPE!) 5047 Eupatorium

Cosup.

usM (3) Herb 2 m a radsid GH Fis-creany silhite det. E.zendiai 5078 . Ipomoea phillomega House Coustunhas Cusco (3) Unie w per thichet along GH road. Calnx purple Corsur F fots beep pink. det. C. Nieggroda $19 f 6$. 
GH $50 \% 9$

Fern

usm (2) terifern is 20 growth.

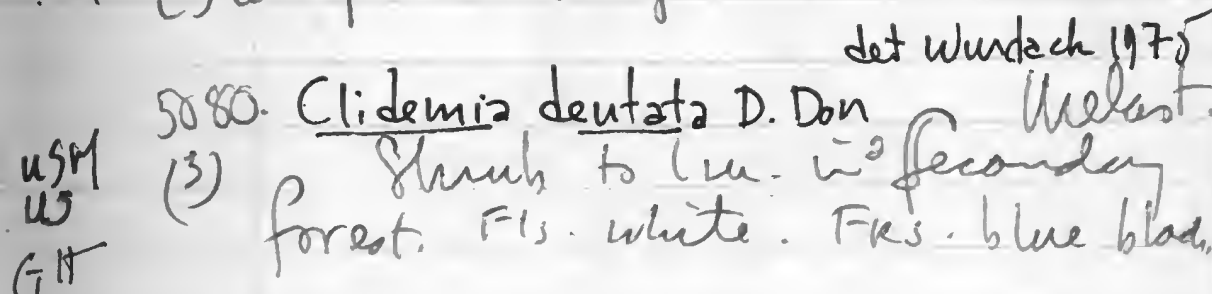

GIt 5881 Angurias?

Cucurb.

$F$ (2) Therb vine is oper thichet. Hyparthir deep trange. Corlla yellow.

USM 5082 Clidania Cordato Cogn. Mulast.

(3) Shrub Im Ovon y a ealyx

Right zellow gree. det Wundack 1975

3083. Ischuosithon

Merent.

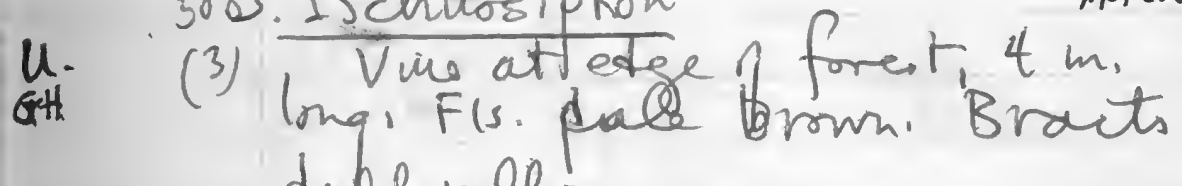
dube yellow.

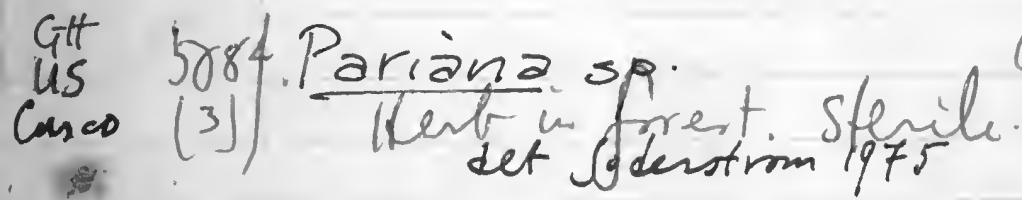

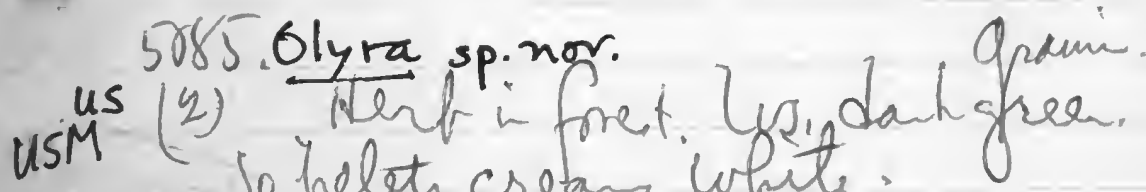

Grom.

det odestron St975 USM - sp helet creang White. 
US 5086 Leandra longicoma Cogn. Melestam USM (3) Itrulo Tm. in ent. Brout It.

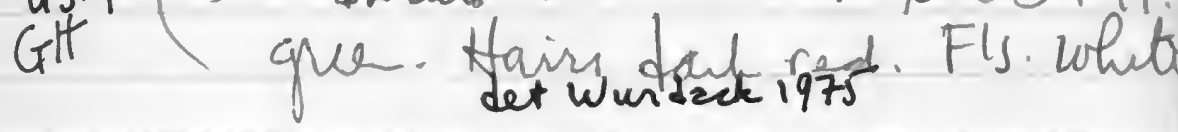
578 f. Tococa caquetana Sprague Melast. GH (1) Shrubls $1.5 n$. Rachis tared. Petals + filament yellow
det Wiendack 1975

a Feb 75 Dept atredaDios: Prov.

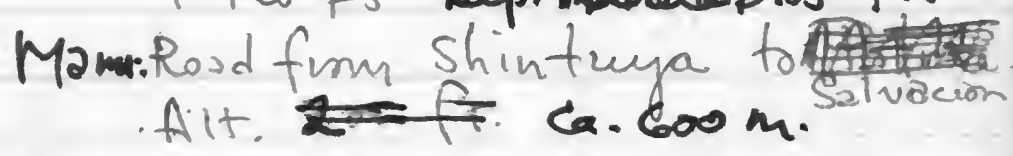

Chzes 5088 . Cleowe spinosa

GH (3) Herb un warh along road.

Cappre. Petals white. Gupophone red. Colyx green.

usm. 559 Gmzalagunic

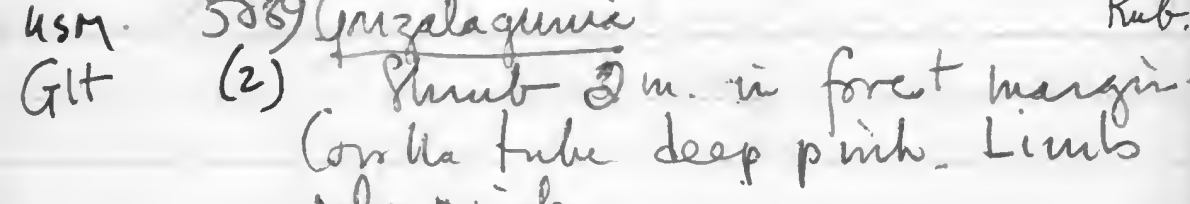
pale pink.

509o. Calathes of.nodosa Rusby Marent $u$ seok Hert 2 m. tall on open (2) distrurbed vadbanh. Brats bed; 2 Lus yellow gree zbove 
pale grengreer beneath, petisle red ovange.

509 Miconia tomentosa (Rich.) Don thelast. usm seed suall tree io low forest mangin, पS It (3) $5 \mathrm{~m}$. Unrine fruit red, ripe fouit purple biach.indach 1975 det

5092. Peperomia

Piperac.

GIt Seniteesin herb in douk forest. Spiker pale green. Frs - yellow- ffeen. US 5093.Encephalosphaera lasiandra Mililor. I Aesuth GH (H) Kenb $2.5 \mathrm{~m}$. ou forest mangi pupt. aloug road. Bracts rose-pint.

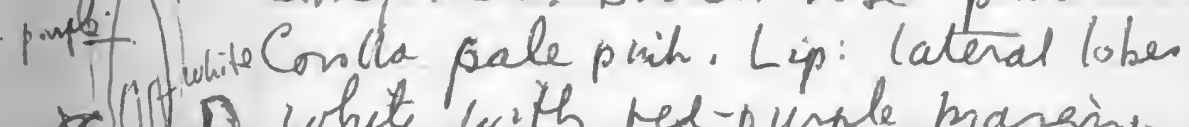
COOS white with red-purple prargin, center lobe red-punple.with 2 white spits nea bas. det. D. Wasshemen 1975 ?

U.

5094. Ctenanthe?

Marentoe. $H$

(4)

Thent $3 \mathrm{~m}$. tall on forest margin in dorb sreen zbove, pole grea beneath. Bracts liqut green. No fis. 
Pilcopata: Villa Cenmen 9 feb.75

Rub.

USM

5045. P Faramea maynensis Spruce ex

GH Shruh $1.5 \mathrm{~m}$. in thichet ${ }^{\text {Rusb }}$ ? in fst.mangin. Rachis pelicil a ovany white, Conlla It blue Roulu in firuct dank blue. Fr. Still green det D. Simpson 1976 .

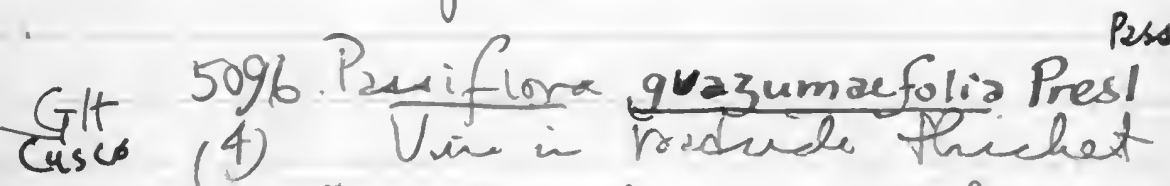
K. Brarts pale grea. Clyx abaxiale palegren; colpx odax sall pefals Creawin whit with soind of ted - purple near base. Inne corose crean White, Stamen spotted purple. Grany crean, SET det Kow 1977.

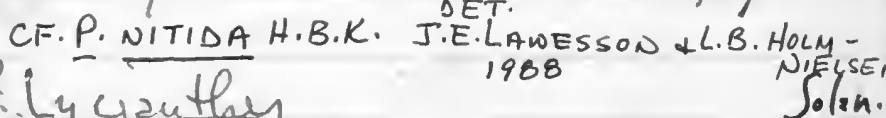

GH 509 \%. Lyciauther

s.t.

$M$

scandent vine in thuchet Con Ua pale violet. Calyx

purple w frut. Fv pale grean

F 5098 Endlicheria dysodanthe (R. +P.) Meg Lauro tacle

Cusco (3) Thee $3-4 \mathrm{~m}$. Pedical + calyx

Git led. Frigraen det. H.w.d. Werff 1986 . 
Guotaviá

5099. Tiar?

leath.

"Iy (i) Syling $2 \mathrm{~m}$. tael in 2 forest.

5100. Cerropia tessmannii

Morec.

US (2) Tive at forest margin, $10 \mathrm{~m}$. tall. Infligreen.

Quitacalzon, wad fiom Pilcopata to Pillawata 37 ro ft 10 fel 75 shady forested quelo raida.

510i. Begonia altaperuvizna. Begon. Cusco (3) Fleshy herf I m. Periaceth $x$ CTH rovary bright red-orauge.

GIt 5102. Ceutropogn

Cempen.

(1) Wers to I u. Corlla pinh.

(Poeppig)Bertth. ex Walp.

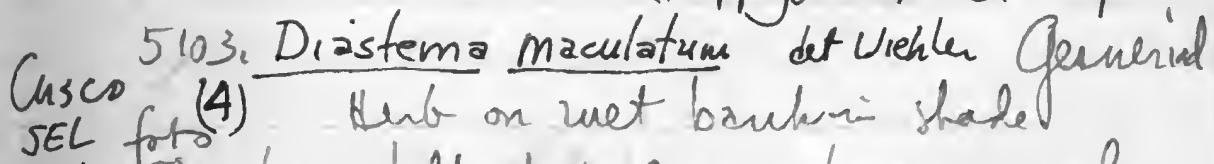

SBN A Les. dall olvis green above, purple

GH beneath. Colyx bmm to green. Con lla tube dill pinh to pupluh, dach purple, datted zith. Lints whete or pale vistet. with inhite inine mariz. 
Preap. GEL 5104 . Besleria cf. divaricata Calx x - orauge. Conlla yellow range. det D. Wasshensen 76

GIV 5105. Steurspermation amomifolium (Poepo) Scosolt

(1) Deer on steep wet bauh? Spathe burm spadic crea,

GH 5106. Floscopa

Connelína.

(1) Hent 0.5 mi lufi..pale yellow ith quen.

GIt 510\%. Centrum

strean in clearnin. Fiels gellow green.

Gt 508. Pilea ut.

(1) Bent I m. Sufl pele green.

GIt 5109. Justicia polygonoides H.B.K. Acenth. Cusco (13) thent $10 \mathrm{~m}$. Qlong strean. 15) Fis Crean White. Wip with aruble vow of spits. det. Wasshersen 1975 
GH 5llo, Cayaponia

Cucurb.

Cusco (4) High dimhin herb vine over.

us. hangma strean. Colnt gredur whit.

Condra pale grea. Frs. 唯h green. shin. $\stackrel{O}{A}$

F? 5111. Glathea

Marant.

Fit (1) Herb $2 \mathrm{~m}$. tall. Lus. yellmish

$H K \leftarrow$ (D) preen 2 brve, ple grey grew benesth. Brocti A. Glea.

Bractesles pale igallon grue. hyeline. Calyx hyaline greeinch Conlla tube croas. 10 bes pale Gilden yellow ked-6rom to wand

Pl. \& Tr. gutiéfers

F 5112 . Chrysochlamys membranacea

Cusco (3) Tree \& u. Tall. Fls. creany

GH yellow. det. C. Niezgode 1982 .

5113 Aphelandra peruviano Wassheura Acauthar

GH (1). Hent 2 m. in deep shade. Bracts light greer. Con lla toee puh

it SII\% Blechnum asplenioides SW.

usc

(4)

Hest on stlep wet radbank. det. K.U.Krames 1975 
Monotagma sp. na.

5115. Merent.

live Low creeping hert on' thep net

USM (A) bodbank among posses.

$H K$ (4) Brasts yelbmish green. Fls.

GH white. det HK, 1978.

3900 ft alt.

GH 5116 . Calcoslario y.engleriana Kränzl.

F (2) Rosdriele herb in luet ploce. Corblla deep yellow. det. U. Molau.

5117. Cestrum Solanize

seed Shrub $3 \mathrm{~m}$. tall in secu

(3) (A) along road. Conlla greewih yellws. Fr. black.

5118 Monopyle macrocarpa Benth. Gesner.

(1) Herls m uet roadbauh. Cegx greer. Corslla tube white, I wib

511. Colditum

Arzc.

(2) Denb in wet radbanh.

- Cusco Spathe whive. Spadic Crean. 
5120. Sobralia violacea

Orchid.

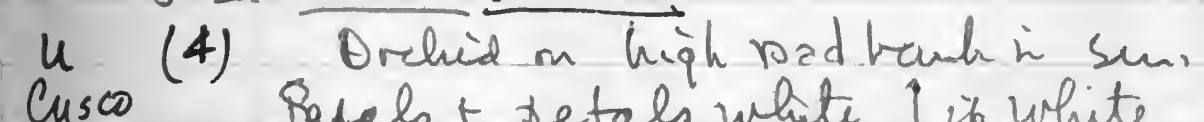
Bepalat setals white. Lip white with agtal spot near tip.

Git 5121. Desinodin

Legum.

Cusco (2) Prostrate herb on rad ent. Fls. pinh.

5122. Miconia theregaus (Bompl.) Cogn. Whelast.

(3) Small tree is per radides US hillsides. Fls. White. det Windack 1975

sit. $6700 \mathrm{ft}$.

= Connajegeriana Uro.

5123. auna leucocarpa Bauché wet (annac.

$u$

(4) Alert $2.5 \mathrm{~m}$. tall on ofen tillaide aloug toad. Brocts green with brown margin. Rachin dunh purplinh b rom. Ovary dorh ted. Calyx crean to pale puiple. Carslla briqut red brange. Staminod + Jtamen Leep traing with red hil spots on adaxial sufaci. $\operatorname{det}$ P.J.M.Maas 1975 . 
Road Peveartumbo to Pileopata. Near Abra Acanacu, Ait. $11400 \mathrm{fi}$

GH $512 \%$. Suphon andra ellipticar (1.+P.) Klotzs $u$ (3) threts I m. tall on mo'st USM hillside. Corrlla waxy, red, Gricac. S. CF. vatKeanus Zahlbr. Sara EDwaros 3/2006 Git 5125. Siphocampylus veteranus E. Wimm. Caupanal. $u$ (4) Hest $2 \mathrm{~m}$. Fall or per medude.

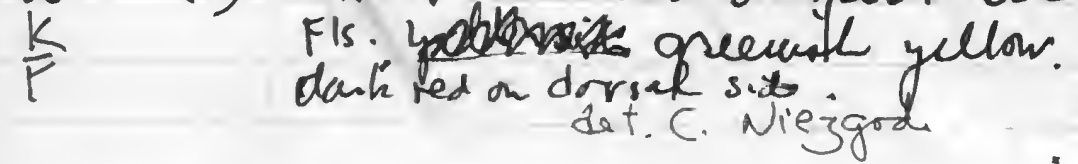

5126. Siphoeampylus elfried ii E. Wimm. Campsnut senb $1.5^{5} \mathrm{~m}$. on rodside.

Cuses celprgrea. Con lla der rose puich Git lohesgren.

det. C. Niezgrida 1976 
Peku: serto. Puno. Pror, Chucuito.

Yunguyo. Lake Titicaca.
18 teb 75 Alt. $3815 \mathrm{~m}$.

5127. Lupinus mutabilis

ECON(2) cult. herb / $/ \mathrm{m}$. tall $\mathrm{fls}$ - deep Col blue-vorlet fornuer purale ot seeds only: F middle wi. "Th yellow sp.t nean base.

\{con 5128. Oxalis tulberosa Molina

Cult Lenb. Stems Jucculent - pale green. Fis. yellow. Tubers

Econ 5 c2lOxzlis tubersa Molina

Cult hert. Stems succulent red. Tubess cream with pinil coloration.

5130. Avena

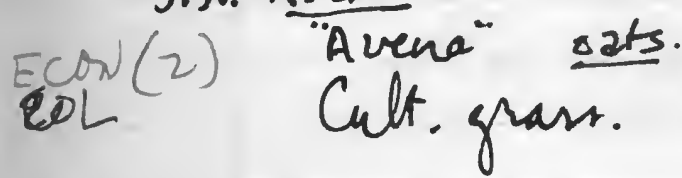

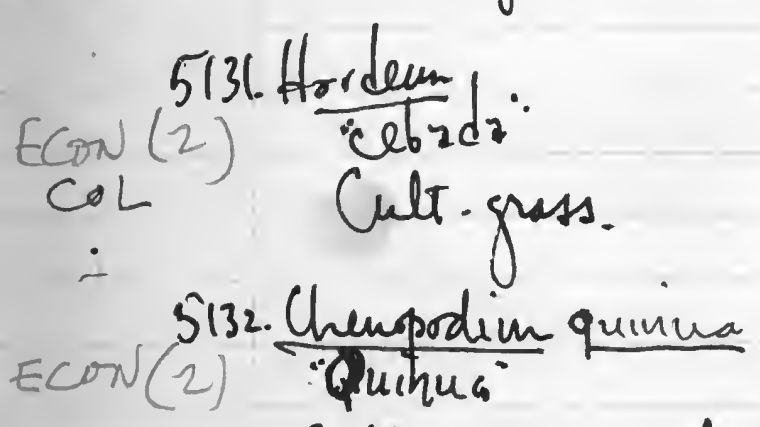

Cult. hert im. Infl.grento reldit 
5133. Calycera pulvinata Remy

GTt, LA Suce. Rowett herb in sand on 1996 . lake shree. Fls. white.

Bolivia: Rept. La Paz. Proimanco Kapaci Above Copa copane. 18 Fel- 1975.

5134. Cantua

$u$ (3) Shrub 4 . . tall on pe $K$ radside. Calp $x$ tark purpler bronge. Corlla dees carmiñe. "cantuta"

GH 5135. Solzunum

BIRM (2) Shrub In. an rodside. Fis. pumple.

Precu: Dept. Puno. Prov. Melgar. Abrade ha Reya. 4300 m.alt.
18 reb 1975 . 18 reb 1975 . coronata Hook. et Arn.

u (4) Cajophora coronstifotio Prest GIt anumprocks. Fls. red-orange. det Kew 1977 . 
Bolivia: Lapto. Paz. $121 \mathrm{Fel} 1975$. valle de la Luna. Alt- $11000 \mathrm{ft}$ 5/3f. Dunalia

GH Spiny shrub im. tack in dry, Mo barren theavily eroded soil. FIs. BirY deep blue-wiolet.

$\operatorname{det}$ A. Huryiger 1976

Bolivis: Desto. La Paz. Prov. Nor Yungas. Between ba Cumbre and Unduavi. 22 fel 75

5138. Solpichroag glandulosa (Hook.) Miers ssp.glandulosa (A) Weah shrub hanging form roch usc (7) walls. Calyt green to purple$F$ nids bronze. Conlla levion yellow in Git 4 bud, becouriy golden yellow, no $\rightarrow$ rendant. det. S. Keel 1983.

$B I R M \rightarrow K$

(A) $\rightarrow$ NY

Below Unduavi. Alt. $10500 \mathrm{ft}$.

E 513\%. Lamerruxia

(3)

slender virgate shrub on steep

GHY roadbauths I $\mathrm{m}$. tall. Colyx finged with deep wine red. Folla scarlet.

Saracha punctata R.NP.

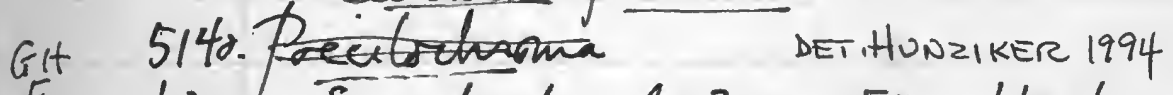

(3) Erect shrub $3 \mathrm{~m}$. FRs. blach.

$804 K$

Mo-live 
Brivia: Dept. La Paz. Prov. Nor Yunges. Road Unduavi to Sacramento. A It. $10,000 \mathrm{ft} .22$ Fet $1975^{\circ}$.

4 5141. Desfontziniez spinosz R. +P.

GH Dscandent Shrub be steep hils id $2 \mathrm{~m}$.

(3) Cahx broun. Corrua tuke light

ved, lobes pale yellow.

Centropogon

5142. Cestroporath brittonianus Zahlb.

Git (2) Nenb 2 u. Fall on rad cut. ovary purple. Caly< loles olvie Corolla tube yellow ish baseed PTBC grotibleasmine red above. Frs. yellow-gree.

Alt. 9200 fo

5143. Spiranthes

fires (1) kenb almong ferunt frasses on wet rosd trank. hff. greenish.

5144 . Lthicularia alpina Jacg.

$u$ (A) Heub on steep wet cliffs a mone

(2) mosses. Epper sepal purpletbtown, locwer pale greas, Spur

A $\rightarrow K$ crean. Cordlat pall Violer with 
pale yellow gethones sput.

Alt.7500 feet.

5145. Feugites mexicana (kunth.) Trin. Gramin. its (2) Crepungthy ou cliff under water fall.

5146. Hedyosmuin racemosum (R.tP.) Solms- Laubact $^{2}$ Glt (D) (2) Treelet $5 \mathrm{~m}$. on open hills ide Spites gremish yellow. det. C.ttie zadg

5147 . Peperonia

$u$ (2) sum. herb. Stems +1 rs. pale Glt green. Sp.her pale quen.

5148 . Miconia op.

holest.

U (3) Treelet $5 \mathrm{~m}$. on ope Lillside.

US YIt Yomggowth t tachis rust.

Buds cheare ci 1975.

us5149. Miconis ef. cordata Triana det wundeck 1775

usM (4) Tree 6 un. On steep ofe us"4 Lillaide Lus. greenzbove, yellor. stand yellow. 
Git $5150.6200 \mathrm{ft}$. Solanum luteo album BIRT 5150. Cuponandxa? - det. A. Vel aff. YO (4) sead Scandent Shants 2 m. tall on of hillsid. Fr. It. rauge.

(5151. Tibouchina or bignyana (Naud) logn. Thelast. Gits (3) Shrub $1.5 \mathrm{~m}$. an ope roadus back. Petalot filaments very pale yellow. Authen go ldew C.H 5152 . Cits 5152 . Phijounter herb on steep roal.
usm (4) cut.

GIt (3) $\frac{\text { Mandevilla }}{\text { Shrub } 0.8 \mathrm{~m} \text {. on rochyout. }}$ USM foto crpeloug royd. Con lla wilite, yellow it throat.

GH 5154 . Alusia Ny (3) infl. yellowist. FNs pole green.

GII 5155 . Miconia ternatifolia Triana tholat. us (3) Thrub 3 m. Young fir. green. 
Alt. $6000 \mathrm{ft}$.

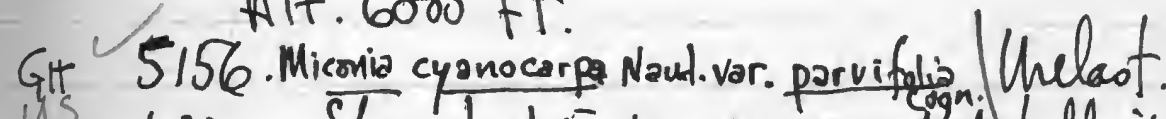

us (3) Shrub $1.5 \mathrm{~m}$. on worded hello ide. Fres-white, det Wurdack 1975

GAt 5157 . Deshodium

creeping suberect herirn rod ditch. red. Conllativiolet.

5158. Misoniz cordata Triana Mulast. uss (3) Taye shrub with spreadi GIt branche. Twigs- rusty brous.

5.59

Oranid.

Ames (D) (i) Epiphyte an wooded hillsidoFis. deep yellow.

Alt. 5000 fr. Nean Corsico.

Anues 5160 . Habenaria

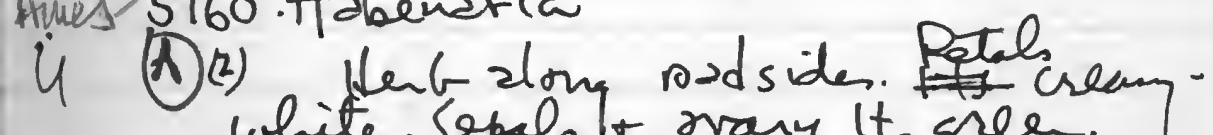
white. Sopelst stany it. green. E galactia speceiosa (DC.) Schum)

u (3) Beadside sherub $1.5 \mathrm{~m}$. Corr had Fit scanlet Calpx lt. 6 mon. 
Betiven yoloso and Ul Choro. A1t. $3500 \mathrm{ft}$.

F S/br. Mabea longifolia(Britton)Pax+K.tbftman Euphan Usoy (3) Shrub $2.5 \mathrm{~m}$. Rachis lights Gitt que. Buds red. det D. Simpson 1976 5163. Miconia minutiflora (Bompl.)DC. Melat. 13) Shruls to treelet 3-5 m. Fis creany white. Fragrause of Lyacinth. $\operatorname{det}$ Wurdack 975 .

11t. $3600 \mathrm{ft}$.

$$
\begin{aligned}
& \text { u } \\
& \text { uss } \\
& \text { EIt }
\end{aligned}
$$

$$
u
$$

USA

G

Mc

5164. Prgonopus tubulosus (DC.) Schum.

(4) Scandent shrub $4.5 \mathrm{~m}$. hangin for diffs. Bracts coarlet. Corlla darh wine red. det D. Simpson 1976 .

5165. Reneehuia racemosa

(5) Lenb on stiet bank in thichet.

2.5 m. toll. Inft basal'; Rachi t bracts ol we gree. Fr. dach red, fier $\{$ calyx reusins orauge. 
Between Puento León and Caranavi. Alt- $2500 \mathrm{ft}$. Along Rro Coraico. $23 \mathrm{Feb} 76$.

Cits Su. Doryopteris
usm spores(3) Fern in steep fuls ide along streame

u 5167. Beqonià

semiflesty hert on steep hillsides sthy stream. Lus. dark gree Fis. crean to pinh.

GH 5168 . Cinchona calisaya Wedd. var josephiana Wedd. usM ( $\uparrow$ ) Shrub $1.5 \mathrm{~m}$. on divy rochy butcrop. Fis. cream fragrant at evening. det. D. Simpson 19:t.

u 5169. Miconia Sessilifolia Naud.

thelast.

US (3) 2-3 m. Tall an dry GH lodbachs. Los. Lack greenzepre, tacony beventh. Pe Aclo yellow, filaments red an thers yellow.
det wurdack 1975 
5170. Enthroxylum eifrifoliam St-thil.

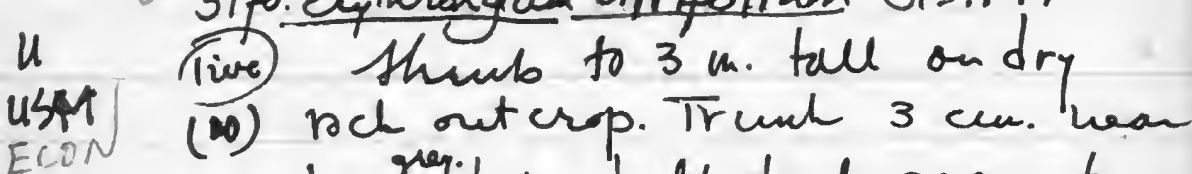
besprilus. Lull dork green to US bellowish-green abore, pale COL u quen beneath with distinct verulet, areolan. regiow indestiuct, no I inea. Hidrito It. brom Frs. rety fer lus. papyraceoum

$51 \%$.

Rubion.

(1) Shrub Im. on dry hichside. seed Rachir a peduncle red. FRs. pungle
prot

Bowiviai Dept. La paz. Prov Larecaja? Roadfurm Caranavi to quanay, Beyond Alcoche. Alt. 4 koo ft.

Gl $517_{2}$. Brunfelsia mire Monachino

$u$ (II) (I) Shrub o. to $1.5 \mathrm{~m}$. tall on USA live steep forested hillside aloug Bikn (E) quebrada, is pastial shade. Ios. * dell, dach greer zbov; It yellowish Inislal green beneath, rubbery. Calyx woburn pale green, tubular-casiparuilate not plicate. Con lla tube very pale 
coilet, Limb pale violet benear, 1t. violet albave with white spot at Inouth.

519. Pariana bicolor Tutin

Panbugaid. usM (5) Give Grass an stees forerted Lidlside albug quebrada. Bracts pirh. spther liqut greer, stemen yellw.

5174. Peperomia

GHt (1) fleshyttenb on steer hichside s long quebrada. Stenes reddich. Spithes yellw. Noisseted orchidiflora (Ru leg)ging.

Git 5175

Hhb $0.5 \mathrm{~m}$ - on bauk fo steam in shade. Fls. orange.

5176. Diastema latiflorum Rushy

(1) semi-fleshy hert ou steep shady hillside 2 lneq que brada. Fls. prate violet. det wiehter 75 ziritareas

Nees + Martius

5177.Erythrochiton brasiliense

U (3) Stt Hub 1-1.5 m. Unbrasom

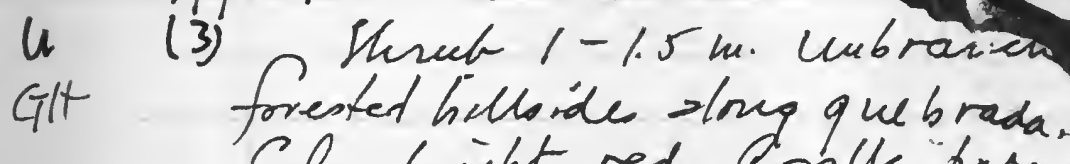

Celpe bright red. Conula pare white. 
Bolivia : Dept. La Paz. Pror. nor-yunges. Beturen Coroico + A yapata. Alt. 6580 ft. 5/48. Hilbenaria

$u$

(8) Herb acumy grasses at edge AMES If rad 6zuh Bracts + racki 1t. green. Calpt spm pale grea.
Pethela t lip whote.

Alt. 5800 feet. $(1760 \mathrm{~m})$.

ECON 5179 . Engthroxy/um Coca

(1) :Cull. shrubt to $1 \mathrm{~m}$. tall in weedy 2-acn field on east facing slope. Soil Rows purpendiculin slope; sued Plants in varionis jages of AfAt F picking.

Coripata. 1760 m.alt.

5180 . Ctenanthe of. Kummeriana Maranta at (1) Cult. heib in hotel garder. L Us. given abore with queystripes ive = PYBG parallel to lat. herves: puphle R.Montyom. beneath. Broct greeuil to bmonish with dach red margun. Fis. ereamy yellom 


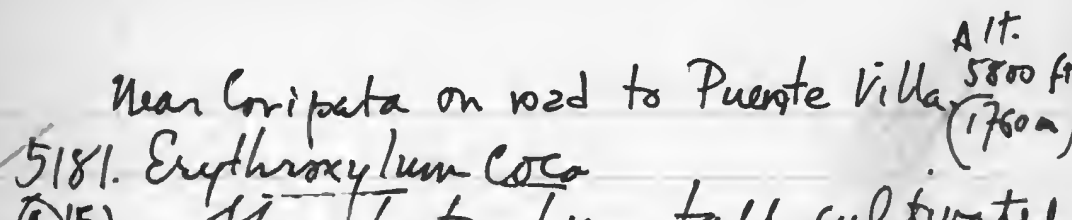

(4) (5) Jisuth to I m. tall cultivated us in hillside field. Lvs. yellnwis green 2 bove, pale green beneath, with poler areslar.

Frs. olive green, turning red at matuity.

5182. Ipomora nil (L.) Roth.

GH (2) Rosdsibe vine in thichet. Conlla deep magenta, white basally. det C. Niezgodal i9f6. BrLTURA: dept La Paz. Prov. Sud yungas. Rit. $6880 \mathrm{ft}$.

Git 5183. Desmodium limense tlook.

(4) The on rodside. Steves, podicel * calpt red. Crolla pale violet.

518t. Groxinia gymnostoma Griseb. Gesuen. (3) Herb on rosdside to I m. Corrlla dere Mieharenta pinl.

5185. Pastiflora subgen. Distephiona, sect. Kermassin

(2) Vine in thichet along rood. Caly $x$ pole green. Corrlla pale blus Corrong in 2 senver, daik quyple at base. Grudod blue twhite'
apically. 
Bolivia: Dept. La Pez. Pru. Noryungas. Alt. 11, 100 feet. Betwee La Cumbre and Unduavi. $24 \mathrm{Fel} 1975$

veds 3

canarioides Unp. tgilg

- 5191. Cajophora buraevi urlo.+-gitg.

$u_{\text {usan }} k_{F}(6)$ Vine in thicket. Fls red-orange. det. 12. Poston $198 \%$

saracha punctata Ricp.

Git 5192. Poecilochroma det. Hunziker 1994 u (6) shrub $1.5 \mathrm{~m}$. tall on oper polsides. Corrlla pudaut, bminish purfle with dall yéllow

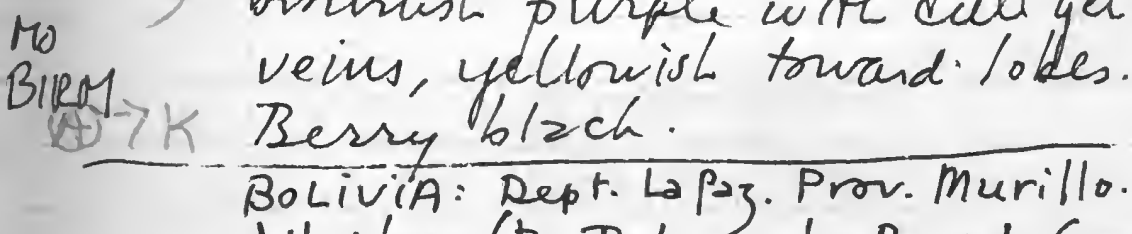

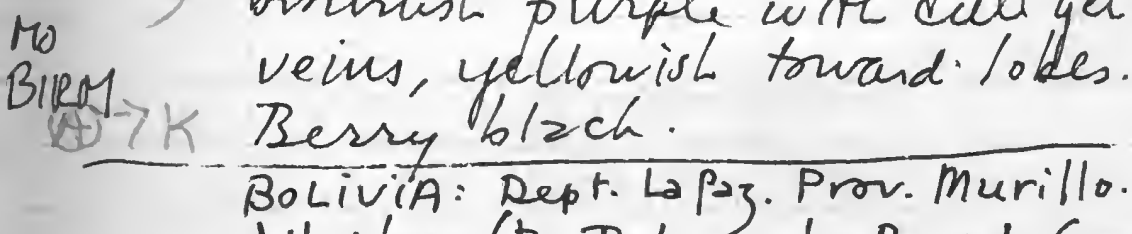
Alt. 14000 ft. Between La P P + La Cumbre

5193. Cajolohora horrita (Brifor)arb.t Gilg u (3) Neit not climbing among rechs on ba rven hillsides. Fis. It red-orange. Hairs urticating.
det. M. Poston 1984 . 
Bolivia: Depto. La Paz. Prov. Murillo. Valle de la Luna. 10,500 feet, $27 \mathrm{Feb} 1975$.

5194. Trichocereun bridgesii (Sam. Dyck) Brittonr ECON Branching columnar eartise to $1.5 \mathrm{~m}$. Tall. No flowers. t.chi Fill Mas. h.v. "achuma" - mescaline Weit San Pedro type.

5195. Euduetinia sp.

$C_{T H}^{(i)}$ shrub $2 \mathrm{~m}$. tall: Fls. rose-pinh. Hypanthin green. det. m. Flores $19 \mathrm{ft}$.

5196. Cuncula ODORATA RUIza PAVON 45M (3) Pasásitic unie on Coupositae. F/s. + FRs. crean,

(A)IK DET.T.BEUZ 1986

5197. Trichocerem bridgesii (Sam-Dyck)Brittont ECON Cult. Shrub $1 \mathrm{~m}$. tall in Cactaris Municipal. Fls. crean white, seeds block.

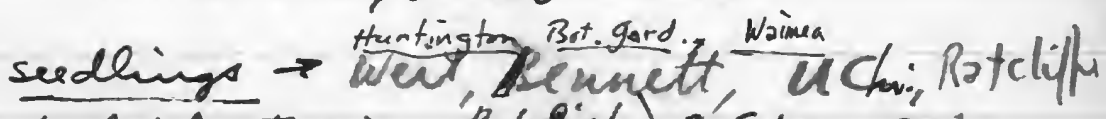
Serds: De Tredici, 'Ratckiffer, C. Schon SEL 
3 MAR 1975.

Bolivia: Dept. La Paz. Alt. $3843 \mathrm{~m}$, Pror. Ingavi. Ruins of Tiwanaku.

CTH 5198. Jaborosasp,

floume.

tosette Lerf on acheological

LSM

BHWTPK site in ofen, distunbed siter.

Fo Frs. green to yellowisl orange

Peru: Depto. Puno. Prov. Chucuito Eepita. $3815 \mathrm{~m}$.

15199. Chenspodim quipen Willd.

Econ (2) Calt herb to $1 \mathrm{~m}$. tall.

dafl. pragental.

5200. Cheurpodium pallidicaule

E(ON) "Ceñihua"

Pult Lent aloug rozdicle in field. Stewer redish. Lower leaves yeltow on redecil. .

Pasu: Dept. Arequipa. ProvrArequipa. Now Paty du road to Juliaca. A A. Cq. $4400 \mathrm{~m}$. GIt 5201. Jaborosa doug razd. Fis. pale goy-blue 
Pren: Dept. Arequipa Prov. Caraveli. Vea Roea Chica. Sea lever.

5202. Dblana

4SM Strawd plant on rochy dunes nean sea. Fis. pele bue. Lus. succulent.] 
Crea leaves

5203.E. coea

Econ (' pound leaver form "Yucay". (ha Convenciö) bought at Andahuaylas, Peku. 10 gan 75. Price: 50 soles $/ 16$.

5204. S. coen

Econ kcosinipata leaver faom kcosinipata Ualley bought of Pilcopzete, Prov! Pacueantambo, Cuzeo. Price' 30 soles/16. ro fer 75 .

5205. S. coes

ECoN 7 pound lus. bought in Cuzee Econ mankt: frm Quillabamba.

$$
\begin{aligned}
& 1 \text { fed } 1975 \text {. } \\
& 50 \text { soles/16. }
\end{aligned}
$$

5206. S. coea.

1 poud leaves bought in

Ear Cuyco market: from Quillabauba. Cuge Verdi" 15 fet 75. 40 soles/lb. Toera $1 \mathrm{sol} / \mathrm{bell}$. 
$520 \%$. E. coen

1 Ib. leaves bruplt is ¿con streetside warket in La Pay.

Sornce: Coripata.

Price: 20 pesos / ib. $28 f e l 65$.

5208. E.cora.

Econ Atreetside monhet i la Paz. Sounce: Coripata:

$$
\text { Prici: } 24 \text { pesos/1b. } 28 \text { feb } 75 \text {. }
$$

PERu: Lima: LA Molma. Jandi-Botanics Cuer. 5209. Erythoxylum novrgranatemse Bht (3) Shrub1.5 m. tall. Same ECON as 4624 . Fls. creamy white. Fruits still green.

(3210. Erythroxylum ulei?

$\mathrm{B} / \mathrm{T}$

ECON

Shrub 1.5 m. cult Sterile. 
5211. Erothroxulum ulei 0.E. Schuly (1) Lhrub / m. tall cultiEcol vatel at quecalouse, $\mathrm{La}$ Molinla Uriversidod Nacional Agrarian,

Collected by Fdgando Machado, at Crisnejos, Dist. Uchiza, Dept. San Martin, Peru. 
Tuamizas de siempre. que siempre te records Rá, ¡Muchas Felicidades! iNo the olvises!..

Polliuqores? \& Frumfelsia australis skippei 1. Quinto cannas (therrich Schäffer), 1869. (Hesperidae)

fly 2. Ornidia obesa (Fabricius). 

$\int_{1}$

is
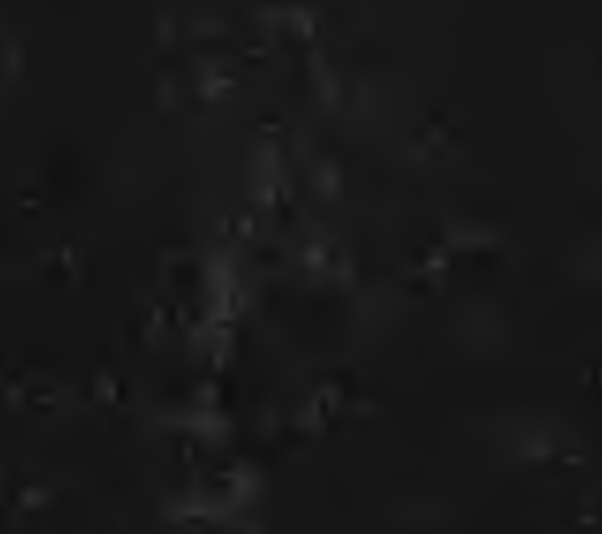

0

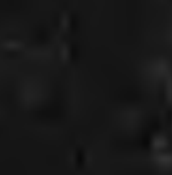

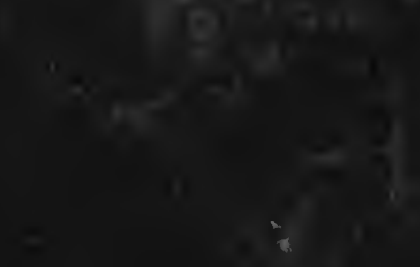

$=$

.

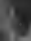

